\title{
Characterization of the self-motion set of the orthogonal spherical mechanism ${ }^{1}$
}

\author{
Albert Castellet, Federico Thomas \\ Institut de Robòtica i Informàtica Industrial (CSIC-UPC), Gran Capità 2-4, 2a planta, 08034 Barcelona, Spain
}

Received 1 October 1997

\begin{abstract}
The information required for solving the inverse kinematic problem associated with any single-loop spatial mechanism is essentially stored in the inverse kinematic solution set of the orthogonal spherical mechanism $\left(S S^{n-3}\right.$ for short). The first part of this paper is devoted to the characterization of $S S^{n-3}$ in terms of its connectivity, singularities and symmetries. The second one focuses on finding simple closed form expressions for its tangent space outside singularities in terms of rotations or rotation axes. Finally, some examples show how the obtained results can be applied to the analysis of spatial mechanisms. (C) 1998 Elsevier Science Ltd. All rights reserved.
\end{abstract}

\section{Introduction}

In a spherical mechanism, any point in a moving body is confined to move within a spherical surface, and all spherical surfaces of motion are concentric. Considering the progress of mechanisms from planar to spherical, and then from spherical to spatial, the spherical mechanisms are an intermediate stage between planar and spatial mechanisms. Actually, plane mechanisms are a special case of spherical mechanisms in which the radius of the sphere extends to infinity.

The orthogonal spherical mechanism with $n$ degrees of freedom is defined as a single-loop kinematic chain consisting of $n$ rotational pairs, so that all axes of rotation meet at a point and any axis is orthogonal to that of the next pair in the chain. A body performing a spherical motion has only three degrees of freedom: rotations about three perpendicular axes. Thus, for $n>3$ this mechanism becomes redundant, so that the set of angles that keep it closed can be seen as a variety of dimension $n-3$ embedded in the $n$-fold torus, $T^{n}$. Herein, this set is called

\footnotetext{
${ }^{1}$ This work has been partially supported by the Spanish CICYT under contract TIC96-0721-C02-01.
} 
the self-motion set of the orthogonal spherical mechanism, $S S^{n-3}$ for short. The self-motion set term is motivated by the fact that any closed path in it leads to a continuous motion of the spherical mechanism which does not require to open it. This paper focuses on the characterization of this set.

The interest of the pursued characterization in the analysis of spatial mechanisms derives from the analysis of the so-called $n$-bar mechanism. The factorization of the loop equation of this particular mechanism into a rotation and a translation equation, shows that, while the solution to the former is directly $S S^{n-3}$, the solution to the latter can be expressed (excluding singular points) in terms of the tangent bundle of $S S^{n-3}$.

Articles published on the analysis and synthesis of spherical mechanisms are quite scattered. The reader is referred to [1] for a compilation and review of some of the important knowledge and techniques so far developed in this branch of the theory of mechanisms.

This paper is structured as follows. Section 2 shows how any kinematic loop equation can be modeled as the loop equation derived from the $n$-bar mechanism. This equation is then factored into a rotation and a translation equation. Next, the solution space of both equations are separately analyzed in Sections 3 and 4, respectively. Since the rotation equation is equivalent to the loop equation of the orthogonal spherical mechanism, Section 3 can also be seen as devoted to the characterization of $S S^{n-3}$. Actually, its dimension, connectness, singularities and symmetries are fully studied in this section. Section 4 can also be seen as a characterization of the tangent space of $S S^{n-3}$, since it is proved that the solution set of the translation equation of the $n$-bar mechanism is provided by the tangent bundle of $S S^{n-3}$. Section 5 contains examples showing the practical interest of most of the obtained results. In Section 6 we summarize conclusions from our study.

\section{The $n$-bar mechanism and a factorization of its loop equation}

The $n$-bar mechanism is a closed single-loop mechanism composed of $n$ links-from now on bars-, each one being orthogonal to the next one. Each bar has two degrees of freedom (d.o.f.): a translational one $\left(d_{i}\right)$, which is the distance along the bar from the previous joint to the next one (it can be negative), and a rotational one $\left(\phi_{i}\right)$, which corresponds to the angle between bar $i+1$ and the plane defined by the two previous bars $(i$ and $i-1)$ measured in the direction of bar $i$ (Fig. 1).

Each bar will have an associated reference frame representing its spatial position and orientation. According to the previous definitions, frame $i+1$ can be expressed in terms of frame $i$ with the transformation $\mathbf{T}\left(d_{i}\right) \mathbf{R}\left(\phi_{i}\right) \mathbf{Z}$, where $\mathbf{T}\left(d_{i}\right)$ stands for a translation along the $x$ axis, $\mathbf{R}\left(\phi_{i}\right)$, a rotation around the $x$-axis and $\mathbf{Z}$, a rotation of $\pi / 2 \mathrm{rad}$ around the $z$-axis.

Then, the loop equation of the $n$-bar mechanism can be expressed as

$$
\prod_{i=1}^{n} \mathbf{T}\left(d_{i}\right) \mathbf{R}\left(\phi_{i}\right) \mathbf{Z}=\mathbf{I}
$$

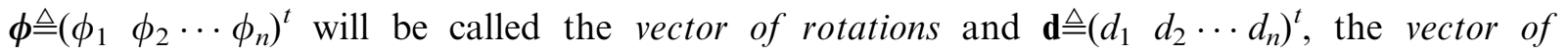
translations. 


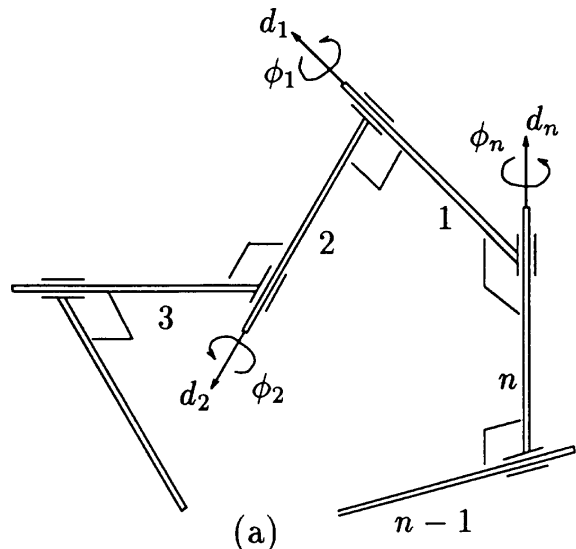

(a)

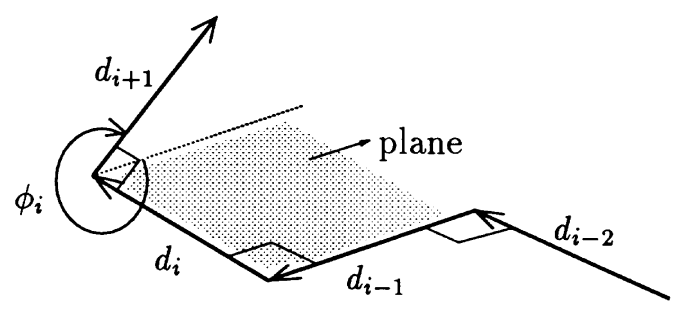

(b)

Fig. 1. The $n$-bar mechanism (a) and definitions of the involved degrees of freedom (b).

Expressing Eq. (1) in terms of homogeneous transforms [2], we get the following matrix equation:

$$
\prod_{i=1}^{n}\left(\begin{array}{cccc}
0 & -1 & 0 & d_{i} \\
\cos \phi_{i} & 0 & -\sin \phi_{i} & 0 \\
\sin \phi_{i} & 0 & \cos \phi_{i} & 0 \\
0 & 0 & 0 & 1
\end{array}\right)=\mathbf{I} .
$$

Remark 1. A mechanism described by the Denavit-Hartenberg parameters $\left(\alpha_{i}, a_{i}, \theta_{i}\right.$ and $\left.t_{i}\right)$ [3] can always be represented by an $\mathrm{n}$-bar mechanism, where

$$
\begin{aligned}
& \left.\begin{array}{c}
\phi_{i}=\alpha_{(i-1) / 2}+\pi \\
d_{i}=a_{(i-1) / 2}
\end{array}\right\} \text { when } i \text { is odd } \\
& \left.\begin{array}{c}
\phi_{i}=\theta_{i / 2}+\pi \\
d_{i}=t_{i / 2}
\end{array}\right\} \text { when } i \text { is even } .
\end{aligned}
$$

We can regard each link of a spatial mechanism as two bars of an $n$-bar mechanism: the odd bars will include the dimensions of each link $\left(\alpha_{i}\right.$ and $\left.a_{i}\right)$ and the even ones will describe the connection between two consecutive links $\left(\theta_{i}\right.$ and $\left.t_{i}\right)$ (Fig. 2). $\pi$ radians have to be added to $\alpha_{i}$ and $\theta_{i}$ because of the used reference frames: while the Denavit-Hartenberg parameter $\theta_{i}$ describes a rotation around the $z$-axis and $\alpha_{i}$, around the $x$-axis, in an $n$-bar mechanism all rotations are described around the $x$-axis.

Three bars are enough to reach any point in three-dimensional space with arbitrary orientation. Then, if the mechanism described by the Denavit-Hartenberg parameters is not closed (e.g. a manipulator), we can always close the associated $n$-bar mechanism with three bars, representing the position of the last bar (the end-effector in a manipulator) with respect to the base. As an example, the Denavit-Hartenberg parameters of the PUMA 560 are given in Table 1. 


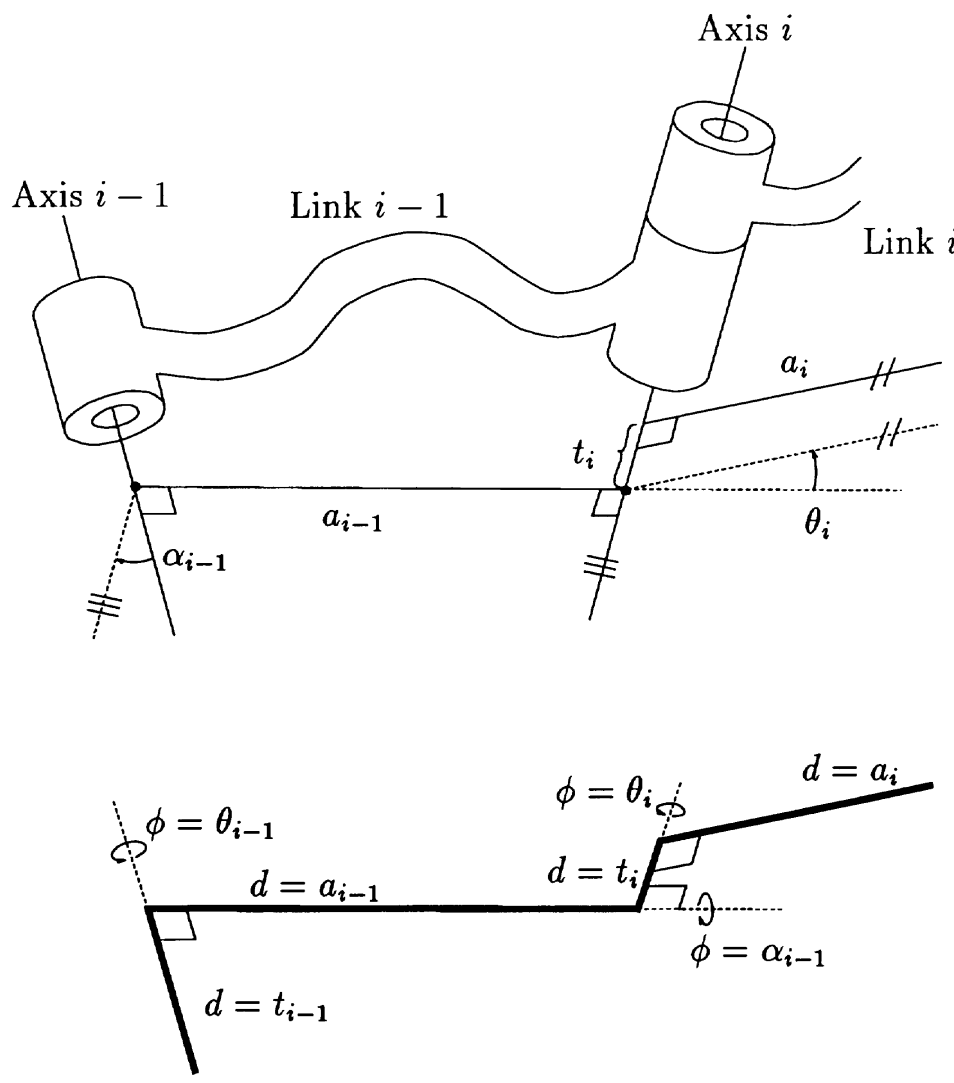

(a)

Fig. 2. The Denavit-Hartenberg parameters of a mechanism (a) and the corresponding $n$-bar mechanism (b).

Then, using Remark 1, the associated $n$-bar mechanism will have the vectors of rotations and translations given in Table 2, where $\phi_{13}, \phi_{14}, \phi_{15}, d_{13}, d_{14}$ and $d_{15}$ are used to represent the end-effector's position with respect to the robot base.

Since any single-loop mechanism can be described by their Denavit-Hartenberg parameters, we conclude that any single-loop mechanism can be described by an $n$-bar mechanism by taking as many bars as needed and restricting some of its degrees of freedom.

Note that the description of a mechanism by an $n$-bar mechanism is not unique. Since the translations are not restricted to be positive, we can always choose between two directions for each bar. This will imply to change the sign of translation $d_{i}$ and also some changes in the neighbouring rotations. These symmetric configurations, which represent the same mechanism, are analyzed in Section 3.5.

The solution of any kinematic equation can be factored into a solution to both its rotation and translation equations. While the solution to the former can be obtained quite easily, the latter leads, in general, to inextricable formulae. This is why this factorization strategy has had very limited practical application in the past, except for some simple problems arising in the field of geometric reasoning [4]. Herein, this strategy plays a fundamental role. 
Table 1

D-H parameters of a PUMA 560

\begin{tabular}{lllll}
\hline$i$ & $\alpha_{i-1}$ & $a_{i-1}$ & $t_{i}$ & $\theta_{i}$ \\
\hline 1 & 0 & 0 & 0 & $\theta_{1}$ \\
2 & $-\pi / 2$ & 0 & 0 & $\theta_{2}$ \\
3 & 0 & $a_{2}$ & $t_{3}$ & $\theta_{3}$ \\
4 & $-\pi / 2$ & $a_{3}$ & $t_{4}$ & $\theta_{4}$ \\
5 & $\pi / 2$ & 0 & 0 & $\theta_{5}$ \\
6 & $-\pi / 2$ & 0 & 0 & $\theta_{6}$ \\
\hline
\end{tabular}

The loop Eq. (1) can be factored into the following two equations [5]:

$$
\mathbf{F}(\boldsymbol{\phi}) \triangleq \prod_{i=1}^{n} \mathbf{R}\left(\phi_{i}\right) \mathbf{Z}=\mathbf{I}
$$

and

$$
\mathbf{T}(\boldsymbol{\phi}, \mathbf{d}) \triangleq \mathscr{N}(\boldsymbol{\phi}) \mathbf{d}=0,
$$

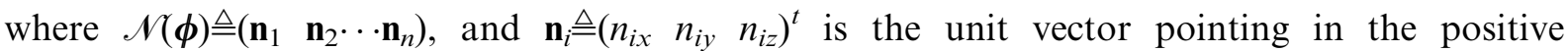
direction of bar $i$ with respect to the first bar of the $n$-bar mechanism. The former Eq. (2) will be called the rotation equation and the latter one Eq. (3), the translation equation. The rotation equation can be extracted directly from the original loop equation by simply removing all translations. It only assures that the final orientation of the chain is the same as the first one, without constraining the translation values. The translation equation, however, involves both translations and rotations. It can also be expressed as

$$
\sum_{i=1}^{n} \mathbf{n}_{i} d_{i}=0 .
$$

It states that the chain really closes, i.e. that the last bar ends at the beginning of the first one without constraining its orientation. This leads to the following remark:

Remark 2. The solutions to both the rotation Eq. (2) and the translation Eq. (3) are the solutions to the loop Eq. (1).

Thus, we can regard the solution to the loop Eq. (1) as the intersection of the solutions to Eqs. (2) and (3). Next, both are separately analyzed.

Table 2

$n$-bar mechanism representing a PUMA 560

\begin{tabular}{llllllllllllllll}
\hline$i$ & 1 & 2 & 3 & 4 & 5 & 6 & 7 & 8 & 9 & 10 & 11 & 12 & 13 & 14 & 15 \\
\hline $\boldsymbol{\phi}$ & $\pi$ & $\theta_{1}+\pi$ & $\pi / 2$ & $\theta_{2}+\pi$ & $\pi$ & $\theta_{3}+\pi$ & $\pi / 2$ & $\theta_{4}+\pi$ & $-\pi / 2$ & $\theta_{5}+\pi$ & $\pi / 2$ & $\theta_{6}+\pi$ & $\phi_{13}$ & $\phi_{14}$ & $\phi_{15}$ \\
$\mathbf{d}$ & 0 & 0 & 0 & 0 & $a_{2}$ & $t_{3}$ & $a_{3}$ & $t_{4}$ & 0 & 0 & 0 & 0 & $d_{13}$ & $d_{14}$ & $d_{15}$ \\
\hline
\end{tabular}




\section{The rotation equation}

There is a spherical kinematic loop associated with any spatial kinematic loop called its spherical indicatrix [6]. Its revolute axes are parallel to those in the corresponding spatial kinematic loop, so that they all intersect at the origin. The rotation Eq. (2) corresponds to the loop equation of this spherical indicatrix which can be simply obtained by removing all translations from the loop Eq. (1).

Remark 3. Any two elements of the diagonal of $\mathbf{F}(\boldsymbol{\phi})$ equaled to 1 are equivalent to considering the whole rotation matrix Eq. (2).

Since $\mathbf{F}(\boldsymbol{\phi})$ is a $3 \times 3$ matrix, the rotation Eq. (2) consists of nine equations. Considering that there are three rotational degrees of freedom in space, it could be expected that there would be three equations representing this information. However, two equations are enough. Let us suppose that $f_{11}$ (the first row-first column element of $\mathbf{F}(\boldsymbol{\phi})$ ) is equaled to 1 . Since $\mathbf{F}(\boldsymbol{\phi})$ is an orthogonal matrix, all row and column vectors are unitary and therefore $f_{12}, f_{13}, f_{21}$ and $f_{31}$ are necessarily 0 . Likewise, if $f_{22}$ is also $1, f_{23}$ and $f_{32}$ will be 0 . Finally, since the determinant of $\mathbf{F}(\boldsymbol{\phi})$ is 1 (it is a product of proper orthogonal matrices), $f_{33}$ has to be 1 .

Lemma 1. The partial derivative of $\mathbf{F}(\boldsymbol{\phi})$ with respect to $\phi_{i}$, for a value of $\boldsymbol{\phi}$ that satisfies the rotation Eq. (2), is a skew-symmetric matrix representing the direction of the $i^{\text {th }}$ bar; that is,

$$
\left.\frac{\partial \mathbf{F}(\boldsymbol{\phi})}{\partial \phi_{i}}\right|_{\mathbf{F}(\boldsymbol{\phi})=\mathbf{I}}=\mathbf{N}_{i}(\boldsymbol{\phi}),
$$

where

$$
\mathbf{N}_{i}(\boldsymbol{\phi}) \triangleq\left(\begin{array}{ccc}
0 & -n_{i z} & n_{i y} \\
n_{i z} & 0 & -n_{i x} \\
-n_{i y} & n_{i x} & 0
\end{array}\right) .
$$

Proof. The partial derivatives of the rotation Eq. (2) with respect to $\phi_{i}$ are straightforward:

$$
\frac{\partial \mathbf{F}(\boldsymbol{\phi})}{\partial \phi_{i}}=\mathbf{A}_{1}^{i-1}(\boldsymbol{\phi}) \mathbf{Q} \mathbf{A}_{i}^{n}(\boldsymbol{\phi}),
$$

where

$$
\mathbf{A}_{k}^{l}(\boldsymbol{\phi}) \triangleq\left\{\begin{array}{cc}
\mathbf{I} & k=l+1 \\
\prod_{j=k}^{l} \mathbf{R}\left(\phi_{j}\right) \mathbf{Z} & k \leq l
\end{array}\right.
$$

and 


$$
\mathbf{Q} \triangleq\left(\begin{array}{ccc}
0 & 0 & 0 \\
0 & 0 & -1 \\
0 & 1 & 0
\end{array}\right)
$$

Since $\mathbf{F}(\boldsymbol{\phi})=\mathbf{A}_{1}^{i-1}(\boldsymbol{\phi}) \mathbf{A}_{i}^{n}(\boldsymbol{\phi})$, if $\boldsymbol{\phi}$ satisfies $\mathbf{F}(\boldsymbol{\phi})=\mathbf{I}$, then

$$
\mathbf{A}_{i}^{n}(\boldsymbol{\phi})=\left(\mathbf{A}_{1}^{i-1}(\phi)\right)^{-1} \text {. }
$$

Moreover, the matrices $\mathbf{A}_{j}^{k}(\boldsymbol{\phi})$ are orthogonal, so that $\left(\mathbf{A}_{j}^{k}(\boldsymbol{\phi})\right)^{-1}=\left(\mathbf{A}_{j}^{k}(\boldsymbol{\phi})\right)^{t}$. Then,

$$
\left.\frac{\partial \mathbf{F}(\boldsymbol{\phi})}{\partial \phi_{i}}\right|_{\mathbf{F}(\boldsymbol{\phi})=\mathbf{I}}=\mathbf{A}_{1}^{i-1}(\boldsymbol{\phi}) \mathbf{Q}\left(\mathbf{A}_{1}^{i-1}(\boldsymbol{\phi})\right)^{t} .
$$

Denoting

$$
\mathbf{A}_{1}^{i-1}(\boldsymbol{\phi}) \triangleq\left(\mathbf{n}_{i} \mathbf{o}_{i} \mathbf{a}_{i}\right) \triangleq\left(\begin{array}{ccc}
n_{i x} & o_{i x} & a_{i x} \\
n_{i y} & o_{i y} & a_{i y} \\
n_{i z} & o_{i z} & a_{i z}
\end{array}\right),
$$

it can be easily shown that

$$
\mathbf{A}_{1}^{i-1}(\boldsymbol{\phi}) \mathbf{Q}\left(\mathbf{A}_{1}^{i-1}(\boldsymbol{\phi})\right)^{t}=\left(\begin{array}{ccc}
0 & -n_{i z} & n_{i y} \\
n_{i z} & 0 & -n_{i x} \\
-n_{i y} & n_{i x} & 0
\end{array}\right),
$$

which proves the lemma.

\section{1. $S S^{n-3}$ and $S M^{n-3}$}

The solution of the rotation Eq. (2) can be seen as a subset of the space formed by the $n$ fold product of the rotational variables, that is, the $n$-torus, $T^{n}$. Eq. (2) has a straightforward geometric interpretation as a spherical polygon, which will be useful later. Let us first recall some concepts. A spherical polygon is a closed figure on the surface of a sphere composed of arcs of great circles (or sides) limited by common points (or vertices). If one assigns a circulating direction to the sides of a spherical polygon, the exterior angle between two adjacent sides is defined as the angle measured from the prolongation of the first side beyond the common vertex, to the second side.

Equation (2) can be interpreted as an $n$-sided spherical polygon. Consider a unit sphere centered at the coordinate origin. As a result of applying successive rotations, the $x$-axis will describe a spherical polygon with sides of length $\pi / 2$ and exterior angles equal to $\phi_{i}$ (Fig. 3).

Note that by varying the angles between two sides of length $\pi / 2$, one can form a triangle whose third side may attain any value between 0 and $\pi$. Therefore, one can arbitrarily fix $n-3$ consecutive variables of an orthogonal spherical mechanism. This leads to a spherical chain with $n-2$ sides that can always be closed using two sides. Moreover, if the resulting angle between these two sides is different from 0 and $\pi$, then there are two solutions corresponding to the two symmetric placements of the two sides on the sphere (Fig. 4). As the angle 


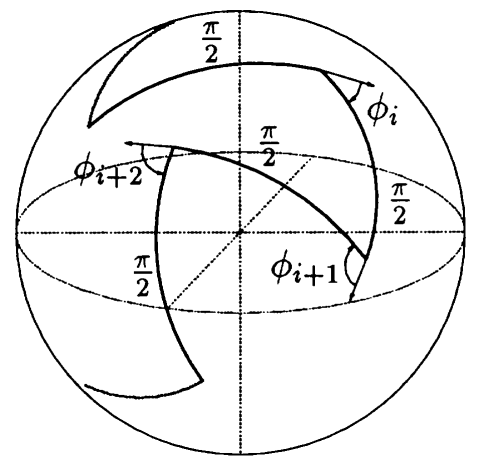

Fig. 3. Spherical polygon generated by the $x$-axis with sides of length $\pi / 2$ and exterior angles equal to $\phi_{i}$.

approaches 0 or $\pi$, the two solution branches fuse into one.

Definition 1 (Self-Motion Set, $S S^{n-3}$ ). The set of points $\boldsymbol{\phi}$ in $\boldsymbol{T}^{\boldsymbol{n}}$ that fulfill the rotation Eq. (2), that is $\left\{\boldsymbol{\phi} \in \boldsymbol{T}^{n} \mid \boldsymbol{F}(\boldsymbol{\phi})=\mathbf{I}\right\}$, will be called the self-motion set, $S S^{n-3}$ for short.

As it has already been mentioned in the introduction, the term self-motion set is motivated by the fact that any trajectory inside this set corresponds to a continuous motion of the corresponding spherical mechanism, which does not require to open it [7].

Proposition. $S S^{n-3}$ is an $(n-3)$-dimensional algebraic variety embedded in $T^{n}$.

Proof. A spherical mechanism becomes redundant for $n>3$. Then, if we choose $n-3$ rotations, we will always be able to find some values for the other three rotations. In general, we cannot fix more than $n-3$ rotations, except for some singular points, as shown in next subsection. Then, since we can locally parameterize $S S^{n-3}$ using $n-3$ parameters, except for some singular points, the self-motion set is an $(n-3)$-dimensional variety.

The self-motion set can be seen as smooth hypersurfaces of dimension $n-3$ possibly intersecting themselves. As it will become clear in next subsection, the stratification of this set leads to a manifold of dimension $n-3$ connected through singular points. See Chapter 1 of

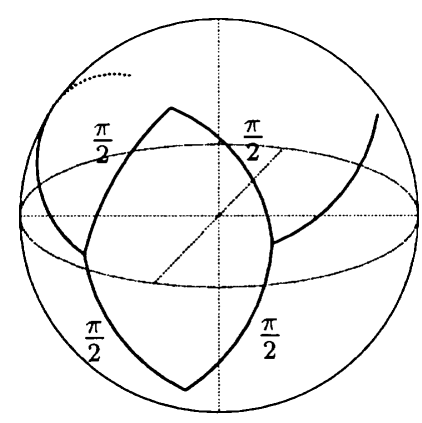

Fig. 4. Two symmetric placements for two sides to close the spherical polygon. 
Part I in [8] for an introduction to the stratification of algebraic sets.

Definition (Self-Motion Manifold, $S M^{n-3}$ ). The self-motion manifold, $S M^{n-3}$, is the manifold resulting from removing all the singularities from $\mathrm{SS}^{n-3}$.

As will be proved next, singular points correspond to those situations in which the $n$-bar mechanism becomes planar, that is when all axes of rotation lie on the same plane. From a topological point of view, removing from $S S^{n-3}$ all these points yields a $(n-3)$-manifold, the self-motion manifold $\left(S^{n-3}\right)$; then, the self-motion set can be alternatively seen as a pseudomanifold, i.e. a punched manifold, since the $(n-3)$-manifold is pinched in a discrete set of points: the singular points.

\subsection{Singularities of $S S^{n-3}$}

The Jacobian of $\mathbf{F}(\boldsymbol{\phi})$ is defined as

$$
\nabla \mathbf{F}(\boldsymbol{\phi}) \triangleq\left(\frac{\partial \mathbf{F}(\boldsymbol{\phi})}{\partial \phi_{1}} \frac{\partial \mathbf{F}(\boldsymbol{\phi})}{\partial \phi_{2}} \cdots \frac{\partial \mathbf{F}(\boldsymbol{\phi})}{\partial \phi_{n}}\right) .
$$

By Lemma 1, the Jacobian in a point $\phi$ of the self-motion set will be

$$
\left.\nabla \mathbf{F}(\phi)\right|_{\phi \in S S}{ }^{n-3}=\left(\mathbf{N}_{1}(\boldsymbol{\phi}) \mathbf{N}_{2}(\boldsymbol{\phi}) \cdots \mathbf{N}_{n}(\boldsymbol{\phi})\right) .
$$

Definition (Global Singularity). Global singularities of $S S^{n-3}$ are defined as those values of $\boldsymbol{\phi}$ for which $\left.\nabla \mathbf{F}(\boldsymbol{\phi})\right|_{\boldsymbol{\phi} \in S S^{n-3}}$ is not of full rank.

If $n$ is even, let us group the rotational variables in couples as follows: $\left(\phi_{1} \phi_{2}\right),\left(\phi_{3} \phi_{4}\right), \cdots$, $\left(\phi_{n-1} \phi_{n}\right)$. Now $a$ will be the number of couples that are $(00), b$ the number of couples $(0 \pi)$ and $c$ the number of couples $(\pi 0)$.

Lemma 2. $\phi=\left(\phi_{1} \phi_{2} \cdots \phi_{n}\right)$ is a global singularity if:

1. $n$ is even

2. $\phi_{i}(\bmod \pi)=0, i=1, \cdots, n$ and

3. $(a+b)$ and $(b+c)$ are both even.

Proof. By analyzing Eq. (6), it is clear that global singularities have to correspond to those situations where all $n$ bars are on a plane. Since an $n$-bar mechanism can degenerate to planar only when $n$ is even, we can conclude that there are no global singularities when $n$ is odd. The second condition of the lemma ensures that the mechanism lies on the plane defined by the first and the last bar. The last condition is a simplified version of the closure Eq. (2) when the second condition holds, inspired in the geometric interpretation of the rotation equation as a spherical polygon. Both provide a necessary and sufficient condition for the $n$-bar mechanism to be in a planar configuration and, hence, for the configuration point to be singular. 
Corollary 1. Global singularities are discrete points of $S S^{n-3}$.

Corollary 2. Global singularities are of corank 1.

Proof. The corank of a singularity is defined as the difference between $\operatorname{rank}\left(\left.\nabla \mathbf{F}(\boldsymbol{\phi})\right|_{\left.\boldsymbol{\phi} \in S M^{n-3}\right)}\right.$ (in a non-singular point) and the rank in the singularity. Note that the rank in a singularity can never be lower than 2 since all the axes of rotation can never be arranged so that they are aligned. Then the corank cannot be greater than 1 .

Corollary 3. When $n$ is odd, $S M^{n-3}=S S^{n-3}$.

Corollary 4. When $n$ is even the number of different singularities is $2^{n-2}$.

Proof. We can choose for the $n-2$ first rotations the values 0 or $\pi$, which leads to $2^{n-2}$ possible combinations. Then, using the above lemma, we can choose one, and only one, of the groups $(00),(0 \pi),(\pi 0),(\pi \pi)$ so that the third condition holds.

\subsection{The connectivity of $S S^{n-3}$ and $S M^{n-3}$}

Note that $S S^{n-3}$ might not be fully connected. Bounds on the number of connected components of the self-motion set are discussed in [7], where it is shown that the self-motion set of a redundant chain can have no more connected components than the maximum number of inverse kinematic solutions of a non-redundant kinematic chain of the same class. A discrete closed-form solution exists for spherical mechanisms with up to three degrees of freedom. For $n=3$, there are two discrete solutions. Therefore, for $n>3$ the self-motion set of a spherical redundant mechanism can have at most two disconnected components.

Lemma 3. $S S^{n-3}$ is a connected subset of $T^{n}$.

Proof. Let us consider the previous interpretation of the rotation equation as a spherical polygon. To prove that $S S^{n-3}$ has a single connected component, it suffices to show that a reference configuration can be reached from every other configuration. When $n$ is even, let the reference configuration be $\phi_{i}=\pi, \forall i=1, \cdots, n$ and when $n$ is odd let it be $\phi_{1}=\phi_{2}=\phi_{3}=\pi / 2$, $\phi_{i}=\pi, \forall i=4, \cdots, n$.

Now, from any initial configuration, one can make the first $n-3$ exterior angles attain sequentially their values at the reference configuration. Thanks to the last two sides, the chain will remain closed throughout the process. When these $n-3$ angles have reached the reference values, the other three angles have to be those corresponding to the reference configuration or its symmetric ones (Fig. 4). If they are the symmetric ones, we can always move the first $n-3$ angles again, so that the two sides corresponding to the last three angles are aligned. Then we move the $n-3$ angles back to the reference configuration, but now bringing the two sides to the right configuration.

Lemma 4. $S M^{n-3}$ is a connected subset of $T^{n}$ when $n>4$. 
Proof. Given the length of the proof, we will only sketch it out. The idea is to link any two points of $S M^{n-3}$ following a path completely contained in $S M^{n-3}$. Any point of $S M^{n-3}$ has at least one $\phi_{i}$ different from 0 or $\pi$. We fix it and make the other angles (except three consecutive ones) attain the values of the other configuration we want to link to it. Then, we make the fixed angle, $\phi_{i}$, attain the corresponding final value. If we were to fall in a singularity, it can be shown that it is avoidable. Finally, we have to check if the three consecutive $\phi_{i}$ used to close the chain are the ones of the final point. If they are the symmetric ones, we have to proceed similarly to the preceding proof.

In Section 5 we will prove that for $n=4, S M^{1}$ is not connected.

\subsection{Parameterizations of $S M^{n-3}$}

The self-motion manifold is an (n-3)-dimensional manifold of class $C^{\infty}$, which can be parameterized by a set of $r=n-3$ independent parameters, $\psi=\left\{\psi_{1}, \psi_{2}, \cdots, \psi_{r}\right\}$, so that it can be locally generated by continuously sweeping $\psi_{i}$ through their ranges. Among all possible parameterizations, we can take $r$ coordinates of the surrounding space $T^{n}$ as local coordinates in the neighborhood of each point $\phi \in S M^{n-3}$. This is, in fact, the implicit function theorem formulated in convenient terms, whose proof can be found in any text book of differential geometry (see, for example, [9]).

Definition (Trivial Parameterization). In particular, we can take $r$ consecutive variables as parameters. This will be called a trivial parameterization.

Let us take a trivial parameterization. Without loss of generality, let the set of parameters $\psi=\left\{\psi_{1}, \psi_{2}, \cdots, \psi_{r}\right\}$ be the first $r$ rotational variables: $\psi_{i}=\phi_{i}(i=1, \cdots, r)$. Then, from Eq. (2) we have

$$
\mathbf{A}(\psi) \triangleq\left(\mathbf{Z A}_{1}^{n-3}(\psi)\right)^{t}=\mathbf{R}\left(\phi_{n-2}\right) \mathbf{Z R}\left(\phi_{n-1}\right) \mathbf{Z R}\left(\phi_{n}\right),
$$

which in general has two solutions for $\phi_{n-2}, \phi_{n-1}$ and $\phi_{n}$ given any proper orthogonal matrix $\mathbf{A}(\psi)$ encompassing all the parameters; namely,

$$
\begin{aligned}
& \phi_{n-2}=\operatorname{atan} 2\left( \pm a_{21}, \mp a_{31}\right) \\
& \phi_{n-1}=\mp \operatorname{acos}\left(-a_{11}\right) \\
& \phi_{n}=\operatorname{atan} 2\left(\mp a_{12}, \mp a_{13}\right),
\end{aligned}
$$

where $a_{i j}$ denotes the element $(i, j)$ of $\mathbf{A}(\psi)$. One solution corresponds to the upper row of signs and the other, to the lower one. Note that the two solutions correspond to the two symmetric 
branches in Fig. 4.

Definition 5 (Singularities of a trivial parameterization). Given a trivial parameterization, the points of $S M^{n-3}$ where $a_{11}= \pm 1$ are called singularities of the parameterization.

In a singularity of a trivial parameterization, when $a_{11}= \pm 1$, Eq. (9) has infinite solutions and it can be shown that they correspond to those situations in which the last three bars are coplanar.

These three equations Eq. (9) almost provide the same information as the rotation Eq. (2); actually, they are equivalent but for the singularities of the chosen parameterization.

Proposition 2. The set of all trivial parameterizations provide an atlas for $\mathrm{SM}^{n-3}$.

Proof. An atlas is a set of parameterizations that covers completely the manifold, which means that any point can be parameterized locally by one of these parameterizations. Any point $\phi^{0}$ in $S M^{n-3}$ has at least an angle $\phi_{i}^{0}$ whose value is different from 0 or $\pi$. Then, we can take a trivial parameterization containing all the angles except $\phi_{i-1}^{0}, \phi_{i}^{0}$ and $\phi_{i+1}^{0}$, which will locally parameterize $S M^{n-3}$ in a neighborhood of $\phi^{0}$.

We can also take $r$ non-consecutive variables as parameters, but the formulation becomes a bit more complex. Actually, the rotation Eq. (2) can be written as

$$
\mathbf{B}(\psi)=\mathbf{R}\left(\phi_{a}\right) \mathbf{C}(\psi) \mathbf{R}\left(\phi_{b}\right) \mathbf{D}(\psi) \mathbf{R}\left(\phi_{c}\right),
$$

where $\phi_{a}, \phi_{b}$ and $\phi_{c}$ are the variables that are not parameters and $\mathbf{B}(\psi), \mathbf{C}(\psi)$ and $\mathbf{D}(\psi)$ are rotation matrices that depend on the parameters. There exist two values for $\phi_{a}, \phi_{b}$ and $\phi_{c}$ that satisfy Eq. (10) if, and only if,

$$
\left(1-c_{11}^{2}\right)\left(1-d_{11}^{2}\right)-\left(b_{11}-c_{11} d_{11}\right)^{2} \geq 0,
$$

where $b_{i j}, c_{i j}$ and $d_{i j}$ denote the elements $(i, j)$ of $\mathbf{B}(\psi), \mathbf{C}(\psi)$ and $\mathbf{D}(\psi)$.

Expressions for $\phi_{a}, \phi_{b}$ and $\phi_{c}$ in terms of the elements of $\mathbf{B}(\psi), \mathbf{C}(\psi)$ and $\mathbf{D}(\psi)$ can be found in [4]:

$$
\begin{aligned}
& \phi_{b}=\operatorname{atan} 2(i h \mp g \omega, g i \pm h \omega) \\
& \phi_{c}=\operatorname{atan} 2\left(e_{13} b_{12}-e_{12} b_{13}, e_{12} b_{12}+e_{13} b_{13}\right) \\
& \phi_{a}=\operatorname{atan} 2\left(f_{32}, f_{22}\right)
\end{aligned}
$$

where 


$$
\begin{aligned}
\omega & =+\sqrt{\left(1-c_{11}^{2}\right)\left(1-d_{11}^{2}\right)-\left(b_{11}-c_{11} d_{11}\right)^{2}} & & \\
g & =c_{12} d_{21}+c_{13} d_{31} & & \mathbf{E}=\mathbf{C R}\left(\phi_{b}\right) \mathbf{D} \\
h & =c_{13} d_{21}-c_{12} d_{31} & & \mathbf{F}=\mathbf{B R}^{t}\left(\phi_{c}\right) \mathbf{E}^{t} \\
i & =b_{11}-c_{11} d_{11} & &
\end{aligned}
$$

In what follows, when we refer a parameterization, we mean a trivial parameterization.

\subsection{Symmetries of $S S^{n-3}$}

The self-motion set of the orthogonal $n$-bar mechanism is highly symmetric. There are symmetric points with respect to any singularity of the parameterization, which correspond to the two solutions of (9).

Definition 6 (Symmetric Points). Given a point $\phi^{0}=\left(\phi_{1} \cdots \phi_{n}\right)^{t} \in S S^{n-3}$, the points

$$
\boldsymbol{\phi}^{i}=\left(\begin{array}{lll}
\phi_{1} \cdots \phi_{i-1}+\pi & -\phi_{i} & \left.\phi_{i+1}+\pi \cdots \phi_{n}\right)^{t} \quad i=1,2, \cdots, n
\end{array}\right.
$$

are also in $S S^{n-3}$ and will be called symmetric points of $\phi^{0}$.

It is obvious that symmetric points are also in $S S^{n-3}$ by analyzing the two solutions of Eq. (9), which correspond to the two symmetric branches in Fig. 4. It will be said that $\phi^{i}$ is obtained from $\phi^{0}$ by applying a symmetry to variable $\phi_{i}$.

Proposition 3. Given two vectors $\boldsymbol{\phi}^{0}=\left(\phi_{1} \cdots \phi_{n}\right)^{t}$ and $\mathbf{d}^{0}=\left(d_{1} \cdots d_{n}\right)^{t}$ that satisfy loop Eq. (1), the vectors

$$
\begin{aligned}
& \boldsymbol{\phi}^{i}=\left(\begin{array}{lll}
\phi_{1} \cdots \phi_{i-1}+\pi & -\phi_{i} & \phi_{i+1}+\pi \cdots \phi_{n}
\end{array}\right)^{t} \text { and } \\
& \mathbf{d}^{i}=\left(d_{1} \cdots d_{i-1} \quad-d_{i} \quad d_{i+1} \cdots d_{n}\right)^{t} \quad i=1,2, \cdots, n
\end{aligned}
$$

also satisfy the loop equation.

Proof. When a symmetry is applied to $\phi_{i}, \mathbf{n}_{i}$ points in the opposite direction. Then, by changing the sign of $d_{i}$, the chain is kept closed.

Lemma 5. Any point on $S S^{n-3}$ has $2^{n-1}$ different symmetric points, except for:

1. singular points, which have $2^{\mathrm{n}-2}-1$ different symmetric points;

2. points that have all its even or odd elements equal to 0 or $\pi$, assuming that $n$ is even, which have $2^{n-1}-1$ different symmetric points.

Proof. It can be seen that the application of two or more symmetries is commutative and that applying a symmetry twice to the same $\phi_{i}$ does not change the point. Thus, the iterative computation of all the symmetries of Definition 6 leads to $2^{n}$ symmetric points; but some of 
these points can be repeated. Applying symmetries to a rotation that is neither 0 nor $\pi$ will change its sign and it is impossible to get again its previous value by applying symmetries to its neighboring rotations. However, if we apply a symmetry to an angle that is 0 or $\pi$, this value does not change and the neighboring ones are incremented by $\pi$. To bring those changed values to their original values, we have to apply a symmetry to its neighboring rotations $(i-2$ and $i+2$ ) and so on. Then, it can be easily shown that the only points that can have repeated symmetric points are those enumerated in this lemma.

Corollary 5. All singular points are symmetric.

Proof. Any symmetric point of a singular point is singular too, since adding $\pi$ or changing the sign of a variable that is 0 or $\pi$ leads to 0 or $\pi$. Then, all singular points have to be symmetric, since there are the same number of different singular points $\left(2^{n-2}\right)$ than different symmetric points of a singular one.

The points of case 2 in Lemma 5 are those corresponding to configurations of mechanisms that have the directions of alternating bars in a plane and the other ones orthogonal to it. This is the case of an $n$-bar mechanism representing a planar mechanism: the odd bars will represent the elements of the planar mechanism and the even bars, of length 0 , will be orthogonal to them representing the axes of rotation of the planar mechanism. Note the difference between an $n$-bar mechanism in a planar configuration (global singularity), where all $n$ bars are contained in a plane, and a planar mechanism represented by an $n$-bar mechanism.

\subsection{Differential approximations of the rotation equation}

In this section, we obtain the Taylor's approximation of $\mathbf{F}(\boldsymbol{\phi}+\Delta \boldsymbol{\phi})$, when $\boldsymbol{\phi}$ is restricted to $S S^{n-3}$, in terms of directions of bars.

Lemma 6. Take a point $\phi \in S S^{n-3}$ and a small perturbation of it, $\boldsymbol{\phi}^{\prime}=\boldsymbol{\phi}+\Delta \boldsymbol{\phi}$. Then, $\phi^{\prime} \in S S^{n-3}$ if, and only if,

$$
\mathscr{N}(\phi) \Delta \phi=0,
$$

which is called the equation of approximation in [10].

Proof. The first order Taylor's expansion of the rotation Eq. (2) around a point $\phi_{0}$ is

$$
\mathbf{F}\left(\phi_{0}+\Delta \phi\right) \simeq \mathbf{F}\left(\phi_{0}\right)+\nabla \mathbf{F}\left(\phi_{0}\right) \Delta \phi .
$$

Given $\phi \in S S^{n-3}, \phi+\Delta \phi$ will also be another point of the self-motion set if

$$
\left.\nabla \mathbf{F}(\boldsymbol{\phi})\right|_{\boldsymbol{\phi} \in S S^{n-3} \Delta \boldsymbol{\phi}=0 .}
$$

Using Eq. (6), we can write this equation in terms of the directions of bars, that is,

$$
\sum_{i=1}^{n} \mathbf{n}_{i} \Delta \phi_{i}=0
$$


which is equivalent to the equation of approximation Eq. (11).

Proposition 4. The $k^{\text {th }}$ derivative of $\mathbf{F}(\boldsymbol{\phi})$ in $\boldsymbol{\phi} \in S S^{n-3}$ is

$$
\left.\frac{\partial^{k} \mathbf{F}(\boldsymbol{\phi})}{\partial \phi_{i_{1}} \partial \phi_{i_{2}} \cdots \partial \phi_{i_{k}}}\right|_{\boldsymbol{\phi} \in S S^{n-3}}=\mathbf{N}_{i}(\boldsymbol{\phi}) \mathbf{N}_{j}(\boldsymbol{\phi}) \cdots \mathbf{N}_{l}(\boldsymbol{\phi})
$$

where $i_{1} \leq i_{2} \leq \cdots \leq i_{k}$.

Proof. By using the same notation in the proof of Lemma 1, the second partial derivative of $\mathbf{F}(\boldsymbol{\phi})$ can be expressed as

$$
\frac{\partial^{2} \mathbf{F}(\boldsymbol{\phi})}{\partial \phi_{i} \partial \phi_{j}}=\mathbf{A}_{1}^{i-1}(\boldsymbol{\phi}) \mathbf{Q} \mathbf{A}_{i}^{j-1}(\boldsymbol{\phi}) \mathbf{Q} \mathbf{A}_{j}^{n}(\boldsymbol{\phi}) .
$$

Since $\mathbf{A}_{i}^{i-1}(\boldsymbol{\phi}) \mathbf{A}_{i}^{j-1}(\boldsymbol{\phi}) \mathbf{A}_{j}^{n}(\boldsymbol{\phi})=\mathbf{I}$ when $\boldsymbol{\phi} \in S S^{n-3}$, we have that

$$
\left.\frac{\partial^{2} \mathbf{F}(\boldsymbol{\phi})}{\partial \phi_{i} \partial \phi_{j}}\right|_{\boldsymbol{\phi} \in S S^{n-3}}=\mathbf{A}_{1}^{i-1}(\boldsymbol{\phi}) \mathbf{Q}\left(\mathbf{A}_{1}^{i-1}(\boldsymbol{\phi})\right)^{t} \mathbf{A}_{1}^{j-1}(\boldsymbol{\phi}) \mathbf{Q}\left(\mathbf{A}_{1}^{j-1}(\boldsymbol{\phi})\right)^{t} .
$$

The second derivatives of $\left.\mathbf{F}(\boldsymbol{\phi})\right|_{\boldsymbol{\phi} \in S S^{n-3}}$ are then the product of the first two derivatives

$$
\left.\frac{\partial^{2} \mathbf{F}(\boldsymbol{\phi})}{\partial \phi_{i} \partial \phi_{j}}\right|_{\boldsymbol{\phi} \in S S^{n-3}}=\mathbf{N}_{i}(\boldsymbol{\phi}) \mathbf{N}_{j}(\boldsymbol{\phi}),
$$

where $i \leq j$. Similarly, higher order partial derivatives in a point of the self-motion set are obtained in terms of the directions of the bars. $\square$

We can express the second order Taylor's approximation of the rotation Eq. (2) for configurations in $S S^{n-3}$ as

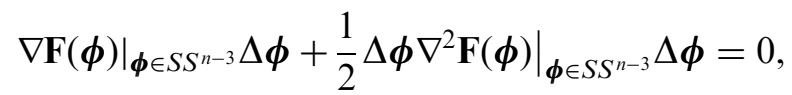

where

$$
\left.\nabla^{2} \mathbf{F}(\phi)\right|_{\phi \in S S^{n-3}} \triangleq\left(\begin{array}{cccc}
\mathbf{N}_{1}^{2}(\phi) & \mathbf{N}_{1}(\phi) \mathbf{N}_{2}(\phi) & \ldots & \mathbf{N}_{1}(\phi) \mathbf{N}_{n}(\phi) \\
\mathbf{N}_{1}(\phi) \mathbf{N}_{2}(\phi) & \mathbf{N}_{2}^{2}(\phi) & \ldots & \mathbf{N}_{2}(\phi) \mathbf{N}_{n}(\phi) \\
\vdots & \vdots & & \vdots \\
\mathbf{N}_{1}(\phi) \mathbf{N}_{n}(\phi) & \mathbf{N}_{2}(\phi) \mathbf{N}_{n}(\phi) & \ldots & \mathbf{N}_{n}^{2}(\phi)
\end{array}\right)
$$

is the Hessian of $\mathbf{F}(\boldsymbol{\phi})$ in a point of the self-motion set.

Higher order approximations could also be obtained in terms of products of the skewsymmetric matrices $\mathbf{N}_{i}(\phi)$. 


\section{The translation equation}

We have seen that the solution space of the rotation equation-excluding global singularities - is a manifold, the self-motion manifold $\left(S M^{n-3}\right)$. The solution space of the translation equation seems to have, however, a much more complicated structure, since it depends on the involved rotations. In this section we will analyze the structure of the solution of the translation equation and its relation with its rotation counterpart.

Proposition 5. The set of solutions $\mathbf{d}=\left(d_{1} d_{2} \cdots d_{n}\right)^{t}$ of the translation Eq. (3)

$$
\mathbf{T}(\boldsymbol{\phi}, \mathbf{d})=\sum_{i=1}^{n} \mathbf{n}_{i} d_{i}=0
$$

is an $(n-3)$-linear variety in $\mathbb{R}^{n}$ if $\phi \in S M^{n-3}$ and an $(n-2)$-linear variety if $\phi$ is a global singularity.

Proof. If $\phi \in S M^{n-3}$, the rank of $\mathscr{N}(\phi)=\left(\mathbf{n}_{1} \mathbf{n}_{2} \cdots \mathbf{n}_{n}\right)$ is 3 , i.e. the mechanism is not planar. Let $\mathbf{n}_{i_{1}}, \mathbf{n}_{i_{2}}, \mathbf{n}_{i_{3}}$ be linear independent. Any choice of $\left\{d_{i} ; i \neq i_{1}, i_{2}, i_{3},\right\}$ determines the three variables $d_{i_{1}}, d_{i_{2}}, d_{i_{3}}$. When $\boldsymbol{\phi}$ is a global singularity, we can only take two linear independent $\mathbf{n}_{i}$, and $n-2$ translations can be taken so that the other two are determined.

Since the solution of the translation equation is a linear variety, it can always be parameterized with $n-3$ or $n-2$ translational variables, $d_{i}$. For example, $\delta_{i}=d_{i}(i=1, \cdots$, $n-3$ ) can be used to parameterize this variety outside of a global singularity if the last three bars are not coplanar.

\subsection{The tangent space of $S M^{n-3}$}

Theorem 1 (Spatial to Spherical Transference). The solution of the translation equation (outside the global singularities of its rotation equation) is the tangent bundle of $S M^{n-3}$, which can be expressed as

$$
\mathbf{d}=\mathbf{K} \lambda, \forall \lambda=\left(\lambda_{1} \cdots \lambda_{r}\right)^{t} \in \mathbb{R}^{n-3},
$$

where

$$
\mathbf{K}=\left[\begin{array}{ccc}
\frac{\partial \phi_{1}}{\partial \psi_{1}} & \cdots & \frac{\partial \phi_{1}}{\partial \psi_{r}} \\
\vdots & & \vdots \\
\frac{\partial \phi_{n}}{\partial \psi_{1}} & \cdots & \frac{\partial \phi_{n}}{\partial \psi_{r}}
\end{array}\right]
$$


and $\psi=\left(\psi_{1} \psi_{2} \cdots \psi_{r}\right)$ is an arbitrary parameterization of $S M^{n-3}$.

Proof. Using Lemma 1, the translation Eq. (15) can be rewritten as

$$
\sum_{i=1}^{n} \frac{\partial \mathbf{F}(\boldsymbol{\phi})}{\partial \phi_{i}} d_{i}=0
$$

or shorter

$$
\nabla \mathbf{F}(\phi) \mathbf{d}=0
$$

which is another version of the translation equation.

The tangent space of an $m$-dimensional variety embedded in an $n$-dimensional space, is defined as the orthogonal complement to the vector space spanned by $n-m$ independent gradient vectors of this variety ([11], p. 80). $\nabla \mathbf{F}(\phi)$ contains nine gradient vectors, but the space spanned by these vectors is only of dimension 3, as explained before. Eq. (17) states that $\mathbf{d}$ has to be orthogonal to these vectors to close the chain and is, therefore, included in the tangent space of $\mathbf{F}(\boldsymbol{\phi})$. The union of the tangent spaces at all points $\phi \in S M^{n-3}$ is called the tangent bundle of $S M^{n-3}$ and is the solution of the translation equation.

Fixing the rotations of the $n$-bar mechanism in a non-planar configuration is equivalent to choosing a point of $S M^{n-3}$. Then, all possible translational solutions, according to Theorem 1 , will be included in the tangent space of the $S M^{n-3}$ at that point (Fig. 5). Since the tangent space is a linear space of dimension $r=n-3$, we can find $r$ vectors as a basis of all solutions for the translational equation.

From Eq. (15), it follows that $\mathbf{d}$ has to be orthogonal to

$$
\mathbf{p}_{x}=\left(n_{1 x} n_{2 x} \cdots n_{n x}\right), \mathbf{p}_{y}=\left(n_{1 y} n_{2 y} \cdots n_{n y}\right) \text { and } \mathbf{p}_{z}=\left(n_{1 z} n_{2 z} \cdots n_{n z}\right) \text {, }
$$

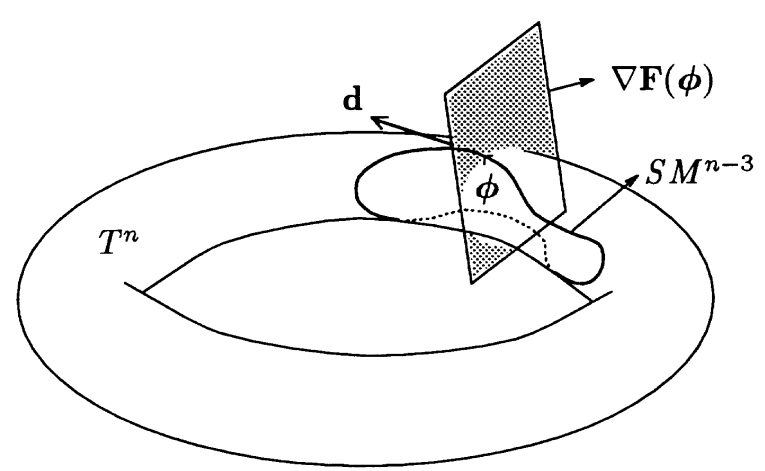

Fig. 5. d must be contained in the tangent space of $S M^{n-3}$ of the corresponding spherical mechanism to keep the $n$ bar mechanism closed. 
which are linearly independent, except for a global singularity. Then, $\mathbf{d}$ has to be included in the orthogonal space of the space defined by $\mathbf{p}_{x}, \mathbf{p}_{y}$ and $\mathbf{p}_{z}$ in $\mathbb{R}^{n}$. We can get a basis of this space by orthonormalizing, using the Gram-Schmidt algorithm, these three vectors with a basis of $\mathbb{R}^{n}$, e.g. the canonical one, $\left.\left[\begin{array}{llll}1 & 0 & \cdots & 0\end{array}\right),\left(\begin{array}{llll}0 & 1 & \cdots & 0\end{array}\right), \cdots \cdots\left(\begin{array}{llll}0 & 0 & \cdots & \cdots\end{array}\right)\right]$. We will get three vectors as a basis of the space generated by $\mathbf{p}_{x}, \mathbf{p}_{y}$ and $\mathbf{p}_{z}$, and $n-3$ vectors as a basis of its orthogonal space.

\subsection{The tangent space in terms of bar directions}

A basis of the tangent space is also the set of vectors $(\partial \phi) /\left(\partial \psi_{i}\right)$, where $i=1, \cdots, r$, as stated in Theorem 1. Next, we obtain this derivatives in terms of the directions of bars.

Theorem 2. The derivatives of $\boldsymbol{\phi}$ in terms of bar directions for a trivial parameterization $\psi_{i}=\phi_{i}$ $(i=1,2, \cdots, r)$ are:

$$
\frac{\partial \phi_{i}}{\partial \psi_{i}}=1 \text { and } \frac{\partial \phi_{i}}{\partial \psi_{j}}=0, i \neq j, \text { for } i, j=1, \cdots, r,
$$

and

$$
\begin{aligned}
\frac{\partial \phi_{n-2}}{\partial \psi_{i}} & =\frac{\left|\mathbf{n}_{i} \mathbf{n}_{n-1} \mathbf{n}_{n}\right|}{\left|\mathbf{n}_{n-2} \mathbf{n}_{n-1} \mathbf{n}_{n}\right|} \\
\frac{\partial \phi_{n-1}}{\partial \psi_{i}} & =\frac{\left|\mathbf{n}_{n-2} \mathbf{n}_{i} \mathbf{n}_{n}\right|}{\left|\mathbf{n}_{n-2} \mathbf{n}_{n-1} \mathbf{n}_{n}\right|} \\
\frac{\partial \phi_{n}}{\partial \psi_{i}} & =\frac{\left|\mathbf{n}_{n-2} \mathbf{n}_{n-1} \mathbf{n}_{i}\right|}{\left|\mathbf{n}_{n-2} \mathbf{n}_{n-1} \mathbf{n}_{n}\right|}
\end{aligned}
$$

Proof. The derivatives of the first $r$ rotations can be straightforwardly calculated:

$$
\frac{\partial \phi_{i}}{\partial \psi_{i}}=1 \text { and } \frac{\partial \phi_{i}}{\partial \psi_{j}}=0, i \neq j
$$

for $i, j=1, \cdots, r$.

To get the derivatives of the variables which are not used as parameters $\left(\phi_{n-2}, \phi_{n-1}\right.$ and $\phi_{n}$ ) with respect to the parameters, the implicit function theorem can be used. To this end, let us define

$$
\varphi_{n-2}(\psi) \triangleq \phi_{n-2}, \varphi_{n-1}(\psi) \triangleq \phi_{n-1}, \varphi_{n}(\psi) \triangleq \phi_{n},
$$

when $\mathbf{F}(\phi)=\mathbf{I}$.

By the implicit function theorem, we can derivate $\mathbf{F}(\psi)$ with respect to any $\psi_{i}$ as follows:

$$
\frac{\partial \mathbf{F}(\boldsymbol{\psi})}{\partial \psi_{i}}=\frac{\partial \mathbf{F}(\boldsymbol{\phi})}{\partial \phi_{i}}+\frac{\partial \mathbf{F}(\boldsymbol{\phi})}{\partial \phi_{n-2}} \frac{\partial \varphi_{n-2}(\boldsymbol{\psi})}{\partial \psi_{i}}+\frac{\partial \mathbf{F}(\boldsymbol{\phi})}{\partial \phi_{n-1}} \frac{\partial \varphi_{n-1}(\boldsymbol{\psi})}{\partial \psi_{i}}+\frac{\partial \mathbf{F}(\boldsymbol{\phi})}{\partial \phi_{n}} \frac{\partial \varphi_{n}(\boldsymbol{\psi})}{\partial \psi_{i}}=0 .
$$

By Lemma 1, 


$$
\mathbf{N}_{n-2} \frac{\partial \varphi_{n-2}(\boldsymbol{\psi})}{\partial \psi_{i}}+\mathbf{N}_{n-1} \frac{\partial \varphi_{n-1}(\psi)}{\partial \psi_{i}}+\mathbf{N}_{n} \frac{\partial \varphi_{n}(\boldsymbol{\psi})}{\partial \psi_{i}}=-\mathbf{N}_{i}
$$

or equivalently,

$$
\mathbf{n}_{n-2} \frac{\partial \varphi_{n-2}(\psi)}{\partial \psi_{i}}+\mathbf{n}_{n-1} \frac{\partial \varphi_{n-1}(\boldsymbol{\psi})}{\partial \psi_{i}}+\mathbf{n}_{n} \frac{\partial \varphi_{n}(\boldsymbol{\psi})}{\partial \psi_{i}}=-\mathbf{n}_{i}
$$

Solving this linear system by using Cramer's rule we get the announced result.

These derivatives have a simple geometric interpretation: $\mathbf{n}_{n-2}, \mathbf{n}_{n-1}$ and $\mathbf{n}_{n}$ constitute a normalized (but non-orthogonal) basis in $\mathbb{R}^{3}$, because the used parameterization is only valid if $\mathbf{n}_{n-2}, \mathbf{n}_{n-1}$ and $\mathbf{n}_{n}$ are not coplanar. It can be easily seen that $-\left(\partial \phi_{n-2}\right) /\left(\partial \psi_{i}\right),-\left(\partial \phi_{n-1}\right) /\left(\partial \psi_{i}\right)$ and $-\left(\partial \phi_{n}\right) /\left(\partial \psi_{i}\right)$ are just the components of $\mathbf{n}_{i}$ when using the basis defined by $\mathbf{n}_{n-2}, \mathbf{n}_{n-1}$ and $\mathbf{n}_{n}$ in $\mathbb{R}^{3}$ (Fig. 6).

Matrix $\mathbf{K}$ in Eq. (1) can now be easily calculated in terms of bar directions. Notice that most of the values of $\mathbf{K}$ are 0 :

$$
\mathbf{K}=\left[\begin{array}{ccc}
1 & \cdots & 0 \\
\vdots & \ddots & \vdots \\
0 & \cdots & 1 \\
-\frac{\left|\mathbf{n}_{1} \mathbf{n}_{n-1} \mathbf{n}_{n}\right|}{\left|\mathbf{n}_{n-2} \mathbf{n}_{n-1} \mathbf{n}_{n}\right|} & \cdots & -\frac{\left|\mathbf{n}_{r} \mathbf{n}_{n-1} \mathbf{n}_{n}\right|}{\left|\mathbf{n}_{n-2} \mathbf{n}_{n-1} \mathbf{n}_{n}\right|} \\
-\frac{\left|\mathbf{n}_{n-2} \mathbf{n}_{1} \mathbf{n}_{n}\right|}{\left|\mathbf{n}_{n-2} \mathbf{n}_{n-1} \mathbf{n}_{n}\right|} & \cdots & -\frac{\left|\mathbf{n}_{n-2} \mathbf{n}_{r} \mathbf{n}_{n}\right|}{\left|\mathbf{n}_{n-2} \mathbf{n}_{n-1} \mathbf{n}_{n}\right|} \\
-\frac{\left|\mathbf{n}_{n-2} \mathbf{n}_{n-1} \mathbf{n}_{1}\right|}{\left|\mathbf{n}_{n-2} \mathbf{n}_{n-1} \mathbf{n}_{n}\right|} & \cdots & -\frac{\left|\mathbf{n}_{n-2} \mathbf{n}_{n-1} \mathbf{n}_{r}\right|}{\left|\mathbf{n}_{n-2} \mathbf{n}_{n-1} \mathbf{n}_{n}\right|}
\end{array}\right]
$$

Then, by Theorem 1,

$$
d_{n-2}=-\sum_{i=1}^{r} \frac{\left|\mathbf{n}_{i} \mathbf{n}_{n-1} \mathbf{n}_{n}\right|}{\left|\mathbf{n}_{n-2} \mathbf{n}_{n-1} \mathbf{n}_{n}\right|} d_{i}
$$

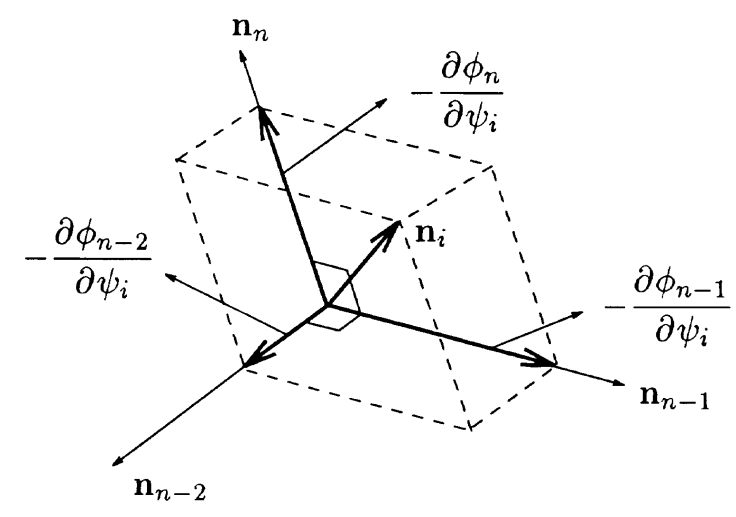

Fig. 6. Geometric interpretation of the derivatives of the variables which are not used as parameters. 


$$
\begin{aligned}
& d_{n-1}=-\sum_{i=1}^{r} \frac{\left|\mathbf{n}_{n-2} \mathbf{n}_{i} \mathbf{n}_{n}\right|}{\left|\mathbf{n}_{n-2} \mathbf{n}_{n-1} \mathbf{n}_{n}\right|} d_{i} \\
& d_{n}=-\sum_{i=1}^{r} \frac{\left|\boldsymbol{n}-\mathbf{2}_{i} \mathbf{n}_{n-1} \mathbf{n}_{i}\right|}{\left|\mathbf{n}_{n-2} \mathbf{n}_{n-1} \mathbf{n}_{n}\right|} d_{i},
\end{aligned}
$$

which is another version of the translation Eq. (15) for non-singular points. This is an important result because of its simplicity (compare it to the development in [12] using spherical trigonometry).

This basis, although comparetively much simpler to get than the one obtained as the orthogonal space of vectors $\mathbf{p}_{x}, \mathbf{p}_{y}$ and $\mathbf{p}_{z}$ in Section 4.1, has a drawback: it is only valid ouside singularities of the parameterization, i.e. when $\left|\mathbf{n}_{n-2} \mathbf{n}_{n-1} \mathbf{n}_{n}\right| \neq 0$. However, this can be easily overcome, since it is always possible to find three non-coplanar consecutive bars, provided that the mechanism is not planar.

From Fig. 1, it can be seen that $\left|\mathbf{n}_{n-2} \mathbf{n}_{n-1} \mathbf{n}_{n}\right|=\sin \phi_{n-1}$. Then the parameterization is valid only when $\sin \phi_{n-1} \neq 0$, which is equivalent to say that the last three bars are not coplanar, as already mentioned. Notice that Eqs. (18) and (19) can be rewritten for any parameterization of $n-3$ variables. The resulting expressions are the same, except that we will have the directions of the three bars that are not parameters in the denominator's determinant.

\subsection{The tangent space in terms of rotations}

Now we will obtain a basis for the tangent space directly from rotations.

Theorem 3. The derivatives of $\frac{\partial \phi}{\partial \phi_{i}}$ for a trivial parameterization $\psi_{i}=\phi_{i}(i=1,2, \cdots, r)$ and the derivatives $\frac{\partial \boldsymbol{d}}{\partial d_{i}}$ for a parameterization $\delta_{i}=d_{i}(i=1,2, \cdots, r)$, can be calculated in terms of rotations as follows:

$$
\left(\begin{array}{c}
\frac{\partial \phi_{n-2}}{\partial \psi_{i}} \\
\frac{\partial \phi_{n-1}}{\partial \psi_{i}} \\
\frac{\partial \phi_{n}}{\partial \psi_{i}}
\end{array}\right)=\left(\begin{array}{c}
\frac{\partial d_{n-2}}{\partial \delta_{i}} \\
\frac{\partial d_{n-1}}{\partial \delta_{i}} \\
\frac{\partial d_{n}}{\partial \delta_{i}}
\end{array}\right)=\mathbf{L}\left(\phi_{n-1}\right)\left(\mathbf{A}_{i}^{n-2}(\boldsymbol{\phi})\right)^{t}\left(\begin{array}{l}
1 \\
0 \\
0
\end{array}\right)
$$

where

$$
\mathbf{L}\left(\phi_{n-1}\right) \triangleq \frac{-1}{\sin \phi_{n-1}}\left(\begin{array}{ccc}
0 & -\sin \phi_{n-1} & \cos \phi_{n-1} \\
\sin \phi_{n-1} & 0 & 0 \\
0 & 0 & 1
\end{array}\right) .
$$

Proof. The translation Eq. (15) can also be written as: 
Table 3

Parametric rotation and translation equations and their derivatives

\begin{tabular}{lll}
\hline & Rotations & Translations \\
\hline Equations & $\phi_{n-2}=\operatorname{atan} 2\left( \pm a_{21}, \mp a_{31}\right)$ & $\left(\begin{array}{c}d_{n-2} \\
d_{n-1} \\
d_{n}\end{array}\right)=\mathbf{L}\left(\phi_{n-1}\right) \sum_{i=1}^{n-3}\left[\left(\mathbf{A}_{i}^{n-2}(\boldsymbol{\phi})\right)^{t}\left(\begin{array}{c}d_{i} \\
0 \\
0\end{array}\right)\right]$
\end{tabular}

Derivatives with respect to $\psi_{i}(\phi)$ $i=1, \cdots, n-3$

$$
\left(\begin{array}{c}
\frac{\partial \phi_{n-2}}{\partial \psi_{i}} \\
\frac{\partial \phi_{n-1}}{\partial \psi_{i}} \\
\frac{\partial \phi_{n}}{\partial \psi_{i}}
\end{array}\right)=\mathbf{L}\left(\phi_{n-1}\right)\left(\mathbf{A}_{i}^{n-2}(\boldsymbol{\psi})\right)^{t}\left(\begin{array}{l}
1 \\
0 \\
0
\end{array}\right)
$$

$$
\left(\begin{array}{c}
\frac{\partial d_{n-2}}{\partial \phi_{i}} \\
\frac{\partial d_{n-1}}{\partial \phi_{i}} \\
\frac{\partial d_{n}}{\partial \phi_{i}}
\end{array}\right)=\mathbf{L}\left(\phi_{n-1}\right) \sum_{k=1}^{i}\left[\left(\mathbf{A}_{k}^{i-1}(\boldsymbol{\phi}) \mathbf{Q} \mathbf{A}_{i}^{n-2}(\boldsymbol{\phi})\right)^{t}\left(\begin{array}{c}
d_{k} \\
0 \\
0
\end{array}\right)\right]
$$

Derivatives with respect to $\phi_{n-2}$, $\phi_{n-1}, \phi_{n}$.

$$
\begin{aligned}
& \left(\begin{array}{c}
\frac{\partial d_{n-2}}{\partial \phi_{n-2}} \\
\frac{\partial d_{n-1}}{\partial \phi_{n-2}} \\
\frac{\partial d_{n}}{\partial \phi_{n-2}}
\end{array}\right)=\mathbf{L}\left(\phi_{n-1}\right) \sum_{i=1}^{n-2}\left[\left(\mathbf{A}_{i}^{n-3}(\boldsymbol{\phi}) \mathbf{Q} \mathbf{A}_{n-2}^{n-2}(\boldsymbol{\phi})\right)^{t}\left(\begin{array}{c}
d_{i} \\
0 \\
0
\end{array}\right)\right] \\
& \left(\begin{array}{l}
\frac{\partial d_{n-2}}{\partial \phi_{n-1}} \\
\frac{\partial d_{n-1}}{\partial \phi_{n-1}} \\
\frac{\partial d_{n}}{\partial \phi_{n-1}}
\end{array}\right)=\mathbf{L}^{\prime}\left(\phi_{n-1}\right) \sum_{i=1}^{n-3}\left[\left(\mathbf{A}_{i}^{n-2}(\boldsymbol{\phi})\right)^{t}\left(\begin{array}{c}
d_{i} \\
0 \\
0
\end{array}\right)\right] \\
& \left(\begin{array}{l}
\frac{\partial d_{n-2}}{\partial \phi_{n}} \\
\frac{\partial d_{n-1}}{\partial \phi_{n}} \\
\frac{\partial d_{n}}{\partial \phi_{n}}
\end{array}\right)=\left(\begin{array}{l}
0 \\
0 \\
0
\end{array}\right)
\end{aligned}
$$

Derivatives with respect to $\delta_{i}$ $i=1, \cdots, n-3$$$
\left(\begin{array}{c}
\frac{\partial d_{n-2}}{\partial \delta_{i}} \\
\frac{\partial d_{n-1}}{\partial \delta_{i}} \\
\frac{\partial d_{n}}{\partial \delta_{i}}
\end{array}\right)=\mathbf{L}\left(\phi_{n-1}\right)\left(\mathbf{A}_{i}^{n-2}(\boldsymbol{\phi})\right)^{t}\left(\begin{array}{l}
1 \\
0 \\
0
\end{array}\right)
$$ 


$$
\begin{aligned}
& \text { Notation } \\
& \mathbf{A}_{j}^{k}(\boldsymbol{\phi}) \triangleq\left\{\begin{array}{l}
\mathbf{I} \\
\prod_{i=j}^{k}\left(\begin{array}{ccc}
0 & -1 & 0 \\
\cos \phi_{i} & 0 & -\sin \phi_{i} \\
\sin \phi_{i} & 0 & \cos \phi_{i}
\end{array}\right)
\end{array}\right. \\
& \mathbf{A}(\boldsymbol{\psi}) \triangleq\left(\begin{array}{ccc}
a_{11} & a_{12} & a_{13} \\
a_{21} & a_{22} & a_{23} \\
a_{31} & a_{32} & a_{33}
\end{array}\right) \triangleq\left(\mathbf{A}_{1}^{n-3}(\boldsymbol{\psi})\right)^{t}\left(\begin{array}{ccc}
0 & 1 & 0 \\
-1 & 0 & 0 \\
0 & 0 & 1
\end{array}\right) \quad \mathbf{Q} \triangleq\left(\begin{array}{ccc}
0 & 0 & 0 \\
0 & 0 & -1 \\
0 & 1 & 0
\end{array}\right) \\
& \mathbf{L}\left(\phi_{n-1}\right) \triangleq \frac{-1}{\sin \phi_{n-1}}\left(\begin{array}{ccc}
0 & -\sin \phi_{n-1} & \cos \phi_{n-1} \\
\sin \phi_{n-1} & 0 & 0 \\
0 & 0 & 1
\end{array}\right) \quad \quad \mathbf{L}^{\prime}\left(\phi_{n-1}\right) \triangleq \frac{1}{\sin ^{2} \phi_{n-1}}\left(\begin{array}{llc}
0 & 0 & 1 \\
0 & 0 & 0 \\
0 & 0 & \cos \phi_{n-1}
\end{array}\right) \\
& \mathbf{T}(\boldsymbol{\phi}, \mathbf{d})=\sum_{i=1}^{n} \mathbf{A}_{1}^{i-1}(\boldsymbol{\phi})\left(\begin{array}{c}
d_{i} \\
0 \\
0
\end{array}\right)=0
\end{aligned}
$$

Applying the implicit function theorem as in Section 4.2 to this equation we get the following expression:

$$
\mathbf{A}_{1}^{i-1}(\boldsymbol{\phi})\left(\begin{array}{l}
1 \\
0 \\
0
\end{array}\right)+\mathbf{A}_{1}^{n-3}(\boldsymbol{\phi})\left(\begin{array}{c}
\frac{\partial d_{n-2}}{\partial \delta_{i}} \\
0 \\
0
\end{array}\right)+\mathbf{A}_{1}^{n-2}(\boldsymbol{\phi})\left(\begin{array}{c}
\frac{\partial d_{n-1}}{\partial \delta_{i}} \\
0 \\
0
\end{array}\right)+\mathbf{A}_{1}^{n-1}(\boldsymbol{\phi})\left(\begin{array}{c}
\frac{\partial d_{n}}{\partial \delta_{i}} \\
0 \\
0
\end{array}\right)=0
$$

Multiplicating by $\left(\mathbf{A}_{1}^{n-3}(\boldsymbol{\phi})\right)^{-1}$ we get

$$
\mathbf{I}\left(\begin{array}{c}
\frac{\partial d_{n-2}}{\partial \delta_{i}} \\
0 \\
0
\end{array}\right)+\mathbf{A}_{n-2}^{n-2}(\boldsymbol{\phi})\left(\begin{array}{c}
\frac{\partial d_{n-1}}{\partial \delta_{i}} \\
0 \\
0
\end{array}\right)+\mathbf{A}_{n-2}^{n-1}(\boldsymbol{\phi})\left(\begin{array}{c}
\frac{\partial d_{n}}{\partial \delta_{i}} \\
0 \\
0
\end{array}\right)=-\left(\mathbf{A}_{i}^{n-3}(\boldsymbol{\phi})\right)^{t}\left(\begin{array}{l}
1 \\
0 \\
0
\end{array}\right) .
$$

This is,

$$
\left(\begin{array}{l}
1 \\
0 \\
0
\end{array}\right) \frac{\partial d_{n-2}}{\partial \delta_{i}}+\left(\begin{array}{c}
0 \\
\cos \phi_{n-2} \\
\sin \phi_{n-2}
\end{array}\right) \frac{\partial d_{n-1}}{\partial \delta_{i}}+\left(\begin{array}{c}
-\cos \phi_{n-1} \\
-\sin \phi_{n-2} \sin \phi_{n-1} \\
\cos \phi_{n-2} \sin \phi_{n-1}
\end{array}\right) \frac{\partial d_{n-1}}{\partial \delta_{i}}=-\left(\mathbf{A}_{i}^{n-3}(\boldsymbol{\phi})\right)^{t}\left(\begin{array}{l}
1 \\
0 \\
0
\end{array}\right)
$$

which leads to the above mentioned expression for the derivatives of the translations. Likewise, 
we get the derivatives for the rotations. Note that $\sin \phi_{n-1} \neq 0$, because it is assumed that we are not in a singularity of the chosen trivial parameterization.

Corollary 6. The translation equations (Eq. (19)) in terms of the rotational variables and their derivatives can be expressed as shown in Table 3.

\section{Examples}

This section contains two examples. The first one is a simple global analysis of the four-bar mechanism, including the stratification of the resulting self-motion set. The second one is a local analysis of a six-bar mechanism in three different situations.

\subsection{Global analysis of the four-bar mechanism}

The loop equation of the four-bar mechanism is:

$$
\prod_{i=1}^{4} \mathbf{T}\left(d_{i}\right) \mathbf{R}\left(\phi_{i}\right) \mathbf{Z}=\mathbf{I} .
$$

The self-motion set will be the set of rotations (points in $T^{4}$ ) that fulfill the rotation equation $\prod_{i=1}^{4} \mathbf{R}\left(\phi_{i}\right) \mathbf{Z}=\mathbf{I}$ that is,

$$
\begin{aligned}
& \left(\begin{array}{cc}
\cos \phi_{2} \cos \phi_{4}+\sin \phi_{2} \cos \phi_{3} \sin \phi_{4} & -\sin \phi_{2} \sin \phi_{4} \\
\sin \phi_{1} \sin \phi_{2} \cos \phi_{4}+\cos \phi_{1} \sin \phi_{3} \sin \phi_{4}-\sin \phi_{1} \cos \phi_{2} \cos \phi_{3} \sin \phi_{4} & \cos \phi_{1} \cos \phi_{3}+\sin \phi_{1} \cos \phi_{2} \sin \phi_{3} \\
\cos \phi_{1} \sin \phi_{2} \cos \phi_{4}+\sin \phi_{1} \sin \phi_{3} \sin \phi_{4}-\cos \phi_{1} \cos \phi_{2} \cos \phi_{3} \sin \phi_{4} & \sin \phi_{1} \cos \phi_{3}+\cos \phi_{1} \cos \phi_{2} \sin \phi_{3}
\end{array}\right. \\
& \cos \phi_{1} \sin \phi_{2} \cos \phi_{4}+\sin \phi_{1} \sin \phi_{3} \sin \phi_{4}-\cos \phi_{1} \cos \phi_{2} \cos \phi_{3} \sin \phi_{4} \quad \sin \phi_{1} \cos \phi_{3}+\cos \phi_{1} \cos \phi_{2} \sin \phi_{3} \\
& \left.\begin{array}{c}
-\cos \phi_{2} \sin \phi_{4}+\sin \phi_{2} \cos \phi_{3} \cos \phi_{4} \\
\sin \phi_{1} \sin \phi_{2} \sin \phi_{4}+\cos \phi_{1} \sin \phi_{3} \cos \phi_{4}-\sin \phi_{1} \cos \phi_{2} \cos \phi_{3} \cos \phi_{4} \\
\cos \phi_{1} \sin \phi_{2} \sin \phi_{4}+\sin \phi_{1} \sin \phi_{3} \cos \phi_{4}-\cos \phi_{1} \cos \phi_{2} \cos \phi_{3} \cos \phi_{4}
\end{array}\right)=\left(\begin{array}{lll}
1 & 0 & 0 \\
0 & 1 & 0 \\
0 & 0 & 1
\end{array}\right) .
\end{aligned}
$$

As stated in Remark 3, we need to solve this system of nine equations only for two equations of the diagonal. Let us take the elements $(1,1)$ and $(2,2)$, which lead to a much simpler system:

$$
\left\{\begin{array}{l}
\cos \phi_{2} \cos \phi_{4}+\sin \phi_{2} \cos \phi_{3} \sin \phi_{4}=1 \\
\cos \phi_{1} \cos \phi_{3}+\sin \phi_{1} \cos \phi_{2} \sin \phi_{3}=1
\end{array} .\right.
$$

To solve it, let us suppose that $\sin \phi_{2} \sin \phi_{4} \neq 0$. Then, from the first equation,

$$
\cos \phi_{3}=\frac{1-\cos \phi_{2} \cos \phi_{4}}{\sin \phi_{2} \sin \phi_{4}} \Longrightarrow\left(\frac{1-\cos \phi_{2} \cos \phi_{4}}{\sin \phi_{2} \sin \phi_{4}}\right)^{2} \leq 1 \text {. }
$$


Changing $\sin ^{2} \phi_{\mathrm{i}}$ for $\left(1-\cos ^{2} \phi_{\mathrm{i}}\right)$, we get

$$
\cos ^{2} \phi_{2}+\cos ^{2} \phi_{4}-2 \cos \phi_{2} \cos \phi_{4}=\left(\cos \phi_{2}-\cos \phi_{4}\right)^{2} \leq 0 \Longrightarrow \phi_{2}= \pm \phi_{4} .
$$

Then, $\cos \phi_{3}= \pm 1$ and from the second equation, $\cos \phi_{1}=\cos \phi_{3}= \pm 1$, leading to the following two solution sets $(\theta \neq 0, \pi)$ :

$$
\boldsymbol{\phi}=\left(\begin{array}{llll}
0 & \theta & 0 & \theta
\end{array}\right)^{t} \text { and } \boldsymbol{\phi}=(\pi \theta \pi-\theta)^{t} .
$$

In a similar way, we can solve the system when $\sin \phi_{2} \sin \phi_{4}=0$, leading to:

$$
\boldsymbol{\phi}=\left(\begin{array}{llll}
\theta & 0 & \theta & 0
\end{array}\right)^{t} \text { and } \boldsymbol{\phi}=\left(\begin{array}{lll}
\theta & \pi & -\theta
\end{array}\right)^{t} .
$$

The stratification of this algebraic set leads to eight strata of dimension 1, whose union is $S M^{1}$, and four of dimension 0 , which are the singular points. As shown in the following diagram, the strata of dimension 1 are connected through those of dimension 0 :

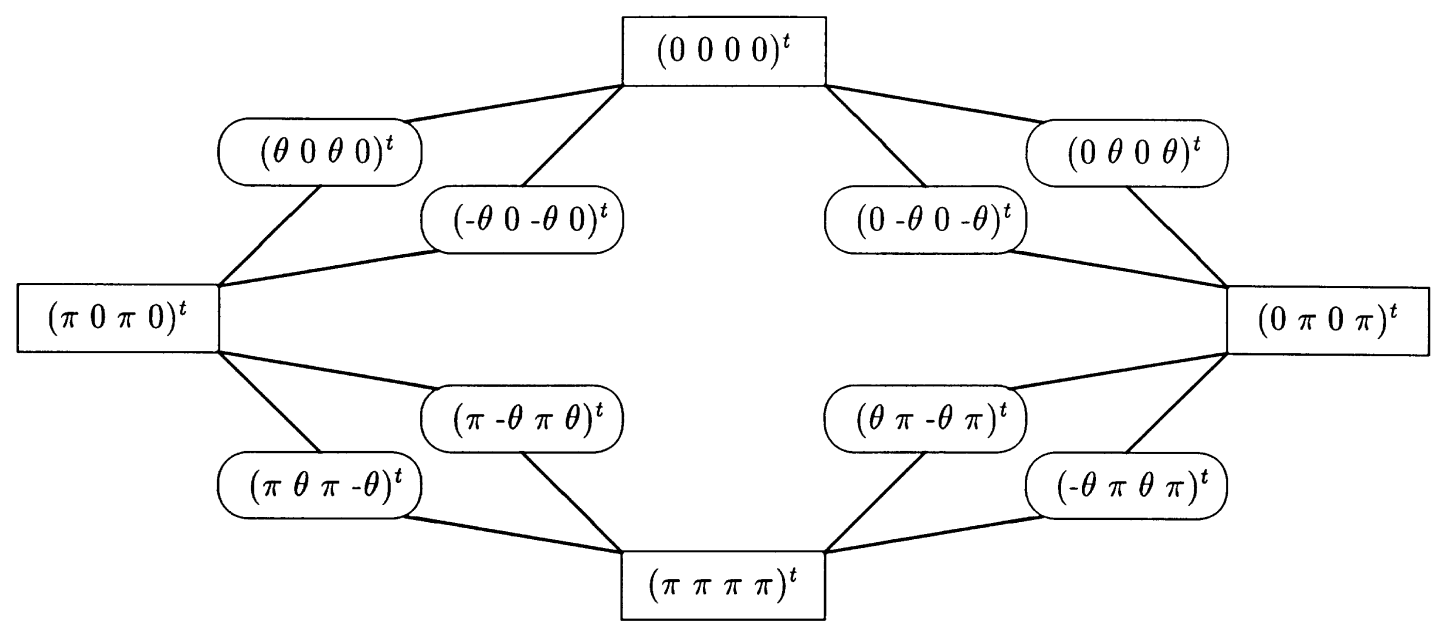

$$
\text { strata of } \operatorname{dim} 0 \quad \text { (singularities) } \quad \text { strata of } \operatorname{dim} 1 \quad(\theta=] 0, \pi[)
$$

Since the number of bars is even, as stated in Corollary 4 , there are $2^{n-2}=4$ singular points, which correspond to the strata of dimension 0 . Notice that $S M^{1}$ is not connected (see Lemma 4).

Observe that all the strata are linear; therefore, their tangent spaces are constant. For example, the tangent space of the upper left strata on the diagram is $\left(\begin{array}{llll}1 & 0 & 1 & 0\end{array}\right)^{t}$. Thus,

$$
\mathbf{d}=\lambda\left(\begin{array}{llll}
1 & 0 & 1 & 0
\end{array}\right)^{t}, \lambda \in \mathbb{R} .
$$

is the only possible solution of the corresponding equation of translations for any point on these strata. For the other strata, the solutions are: 
$\mathbf{d}=\lambda\left(\begin{array}{llll}0 & 1 & 0 & 1\end{array}\right)^{t}$ upper right strata

$\mathbf{d}=\lambda\left(\begin{array}{llll}1 & 0 & -1 & 0\end{array}\right)^{t}$ lower left strata

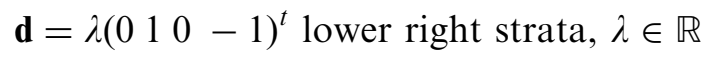

\subsection{Local analysis of the six-bar mechanism}

The loop equation of the six-bar mechanism is

$$
\prod_{i=1}^{6} \mathbf{T}\left(d_{i}\right) \mathbf{R}\left(\phi_{i}\right) \mathbf{Z}=\mathbf{I} .
$$

The loop equation of its spherical indicatrix (the rotation equation) is then

$$
\prod_{i=1}^{6} \mathbf{R}\left(\phi_{i}\right) \mathbf{Z}=\mathbf{I} .
$$

In Section 3 we pointed out that, although this expression consists of nine equations, the dimension of the self-motion set is only $n-3=3$. Here, the configuration space is the torus of dimension $6, T^{6}$, and $S S^{3}$ is a sub-set of $T^{6}$ of dimension 3, with some singular points.

The global singularities correspond to the configurations in which the mechanism is planar. If the number of bars were odd, there would be no global singularities. Since it is even, there are $2^{n-2}=16$ global singularities, which are the points that fulfill conditions (Eq. (7)); that is,

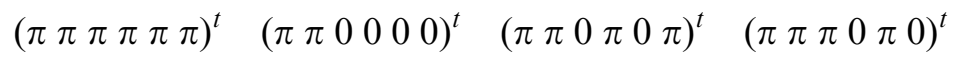

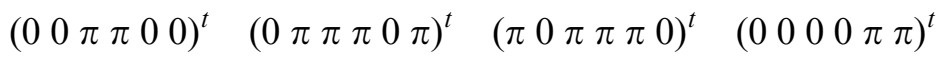

$$
\begin{aligned}
& \begin{array}{lllllll}
(0 \pi 0 \pi \pi \pi)^{t} \quad(\pi 0 \pi 0 \pi \pi)^{t} \quad(000 \pi \pi 0)^{t} \quad(00 \pi 00 \pi)^{t} & (00000
\end{array} \\
& \left.\begin{array}{lllllllllll}
(0 \pi & 0 & 0 & \pi & 0
\end{array}\right)^{t} \quad\left(\begin{array}{llllllll}
0 & \pi & \pi & 0 & 0 & 0
\end{array}\right)^{t} \quad\left(\begin{array}{lllllll}
\pi & 0 & 0 & 0 & 0 & \pi
\end{array}\right)^{t} \quad\left(\begin{array}{lllllll}
\pi & 0 & 0 & \pi & 0 & 0
\end{array}\right)^{t}
\end{aligned}
$$

Notice that any of these singularities can be obtained one from any other by applying symmetries (Corollary 5).

Excluding these points, the set of trivial parameterizations consisting of three consecutive variables provide an atlas for the whole self-motion set (Proposition 2).

\subsubsection{Finding a general solution}

Now, let us suppose that three rotations and two translations are known: $\phi_{1}=30^{\circ}, \phi_{2}=45^{\circ}$, $\phi_{3}=90^{\circ}, d_{2}=7$ and $d_{3}=2$. We look for the values of $\phi_{4}, \phi_{5}, \phi_{6}, d_{1}, d_{4}, d_{5}$ and $d_{6}$ that close the mechanism (Fig. 7).

Since only three rotations are unknown, they can take only two values, corresponding to the two symmetric closures of the spherical polygon (Fig. 4). 


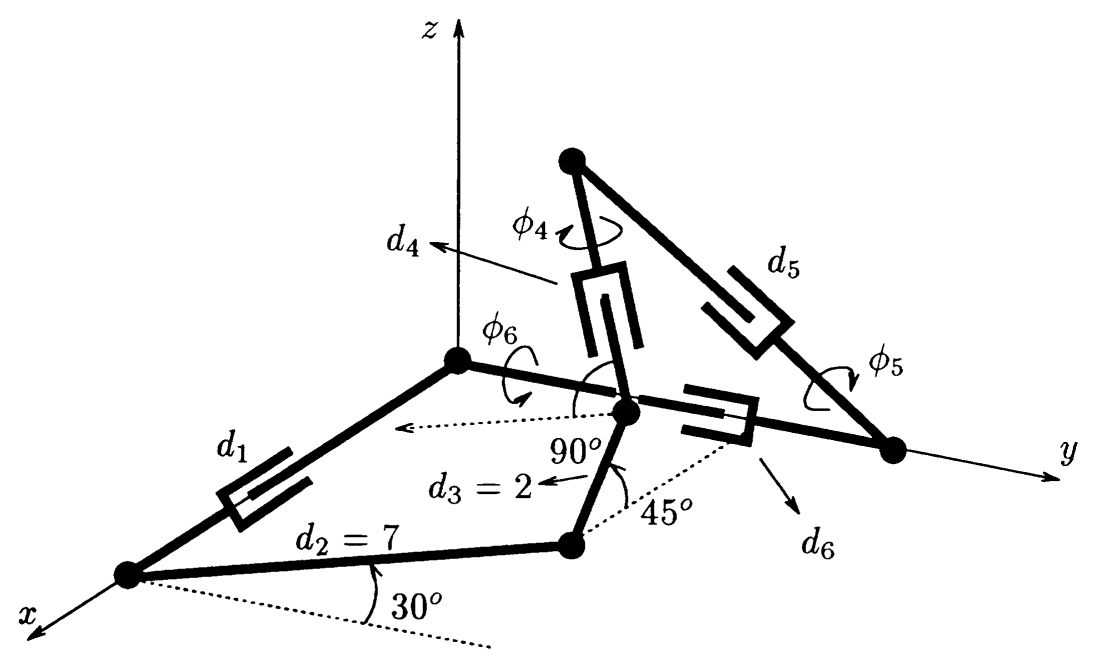

Fig. 7. The six-bar mechanism of the example.

Let us express loop Eq. (20) as in Eq. (8):

$$
\mathbf{R}\left(\phi_{4}\right) \mathbf{Z R}\left(\phi_{5}\right) \mathbf{Z R}\left(\phi_{6}\right)=\left(\mathbf{Z R}\left(\phi_{1}\right) \mathbf{Z R}\left(\phi_{2}\right) \mathbf{Z R}\left(\phi_{3}\right) \mathbf{Z}\right)^{-1}=\left(\begin{array}{ccc}
\frac{\sqrt{2}}{4} & \frac{\sqrt{2}}{2} & \frac{\sqrt{3 / 2}}{2} \\
-\frac{\sqrt{2}}{4} & \frac{\sqrt{2}}{2} & -\frac{\sqrt{3 / 2}}{2} \\
-\frac{\sqrt{3}}{2} & 0 & \frac{1}{2}
\end{array}\right) .
$$

We can calculate $\phi_{4}, \phi_{5}$ and $\phi_{6}$ using Eq. (9)

$$
\begin{aligned}
& \phi_{4}=\operatorname{atan} 2\left( \pm \frac{\sqrt{2}}{4}, \pm \frac{\sqrt{3}}{2}\right)=\left\{\begin{array}{c}
-22.21^{\circ} \\
157.79^{\circ}
\end{array}\right. \\
& \phi_{5}=\mp \operatorname{acos}\left(-\frac{\sqrt{2}}{4}\right)=\mp 110.70^{\circ} \\
& \phi_{6}=\operatorname{atan} 2\left(\mp \frac{\sqrt{2}}{2}, \mp \frac{\sqrt{3 / 2}}{2}\right)=\left\{\begin{array}{r}
-130.89^{\circ} \\
49.11^{\circ}
\end{array} .\right.
\end{aligned}
$$

Note that $\phi_{4}^{1}=\phi_{4}^{0}+\pi, \phi_{5}^{1}=-\phi_{5}^{0}$ and $\phi_{6}^{1}=\phi_{6}^{0}+\pi$ as stated in Section 3.5 (superindexes 0 and 1 denote the two different solutions). We arbitrarely take the first solution, since we can always get the second one as the symmetric of the first one. 
Now, since

$$
\mathbf{n}_{i}=\left[\prod_{j=1}^{i-1} \mathbf{R}\left(\phi_{j}\right) \mathbf{Z}\right]\left(\begin{array}{l}
1 \\
0 \\
0
\end{array}\right),
$$

we can calculate the directions of the six bars:

$$
\begin{aligned}
& \mathbf{n}_{1}=\left(\begin{array}{lll}
1 & 0 & 0
\end{array}\right)^{t} \quad \mathbf{n}_{2}=\left(\begin{array}{lll}
0 & \frac{\sqrt{3}}{2} & \frac{1}{2}
\end{array}\right)^{t} \quad \mathbf{n}_{3}=\left(\begin{array}{lll}
-\frac{\sqrt{2}}{2} & -\frac{\sqrt{2}}{4} & \sqrt{3 / 2} 2
\end{array}\right)^{t} \\
& \mathbf{n}_{4}=\left(\begin{array}{lllll}
\frac{\sqrt{2}}{2} & -\frac{\sqrt{2}}{4} & \frac{\sqrt{3 / 2}}{2}
\end{array}\right)^{t} \quad \mathbf{n}_{5}=\left(\begin{array}{llll}
\frac{\sqrt{3}}{\sqrt{7}} & 0 & -\frac{2}{\sqrt{7}}
\end{array}\right)^{t} \quad \mathbf{n}_{6}=\left(\begin{array}{llll}
0 & -1 & 0
\end{array}\right)^{t} .
\end{aligned}
$$

We can now obtain a basis of the tangent space of $S M^{3}$ in $\phi^{0}$ as the orthogonal space to vectors $\mathbf{p}_{x}, \mathbf{p}_{y}$ and $\mathbf{p}_{z}$ as explained in Section 4.1. Using the Gram-Schmidt method to orthonormalize these three vectors with the canonical basis of $\mathbb{R}^{6}$, we get the following basis for the tangent space of the self-motion manifold in $\phi^{0}$ :

$$
\mathbf{d}=\left(\begin{array}{c}
d_{1} \\
7 \\
2 \\
d_{4} \\
d_{5} \\
d_{6}
\end{array}\right)=\left[\begin{array}{ccc}
0.7483 & 0 & 0 \\
-0.0926 & 0.6682 & 0 \\
0.3024 & 0.0327 & 0.7000 \\
-0.5292 & -0.2291 & 0.1000 \\
-0.2449 & 0.2828 & 0.6481 \\
0 & 0.6481 & -0.2828
\end{array}\right]\left(\begin{array}{l}
\lambda_{1} \\
\lambda_{2} \\
\lambda_{3}
\end{array}\right) .
$$

Solving this linear system, the set of solutions is:

$$
\begin{aligned}
& d_{1}=4.04-1.71 \lambda \\
& d_{4}=-5.43+1.38 \lambda \\
& d_{5}=1.85+1.12 \lambda \\
& d_{6}=7.27-0.49 \lambda,
\end{aligned}
$$

where $\lambda \in \mathbb{R}$.

Assuming that we want to set $d_{5}$ to 0 , the vectors of rotations and the vector of translations that close the mechanism are

$$
\boldsymbol{\phi}^{0}=\left(\begin{array}{llllll}
30 & 45 & 90 & -22.21 & -110.70 & -130.89
\end{array}\right)^{t}
$$




$$
\mathbf{d}^{0}=\left(\begin{array}{llllll}
6.87 & 7 & 2 & -7.72 & 0 & 8.08
\end{array}\right)^{t} .
$$

By applying a symmetry to $\phi_{5}$, as described in Section 4.3, we obtain another solution which corresponds to the second solution of Eq. (21). $\phi_{4}$ and $\phi_{6}$ are incremented by $\pi$ and $\phi_{5}$ and $d_{5}$ change their signs:

$$
\begin{aligned}
\boldsymbol{\phi}^{1} & =\left(\begin{array}{llllll}
30 & 45 & 90 & 157.79 & 110.70 & 49.11
\end{array}\right)^{t} \\
\mathbf{d}^{1} & =\left(\begin{array}{llllll}
6.87 & 7 & 2 & -7.72 & 0 & 8.08
\end{array}\right)^{t} .
\end{aligned}
$$

We could have also used a parameterization to get a basis for the tangent space of $S M^{3}$ as described in Section 4.2. Taking $\phi_{1}, \phi_{2}$ and $\phi_{3}$ as parameters, using Eq. (18) we have

$$
\begin{array}{lll}
\frac{\partial \phi_{4}}{\partial \psi_{1}}=-\frac{\left|\mathbf{n}_{1} \mathbf{n}_{5} \mathbf{n}_{6}\right|}{\left|\mathbf{n}_{4} \mathbf{n}_{5} \mathbf{n}_{6}\right|}=-0.8081 & \frac{\partial \phi_{5}}{\partial \psi_{1}}=-\frac{\left|\mathbf{n}_{4} \mathbf{n}_{1} \mathbf{n}_{6}\right|}{\left|\mathbf{n}_{4} \mathbf{n}_{5} \mathbf{n}_{6}\right|}=-0.6547 & \frac{\partial \phi_{6}}{\partial \psi_{1}}=\cdots=0.2857 \\
\frac{\partial \phi_{4}}{\partial \psi_{2}}=-0.3499 & \frac{\partial \phi_{5}}{\partial \psi_{2}}=0.3780 & \frac{\partial \phi_{6}}{\partial \psi_{2}}=0.9897 \\
\frac{\partial \phi_{4}}{\partial \psi_{3}}=0.1429 & \frac{\partial \phi_{5}}{\partial \psi_{3}}=0.9258 & \frac{\partial \phi_{6}}{\partial \psi_{3}}=-0.4041 .
\end{array}
$$

Then a basis of the tangent space is

$$
\mathbf{K}=\left[\begin{array}{ccc}
1 & 0 & 0 \\
0 & 1 & 0 \\
0 & 0 & 1 \\
-0.8081 & -0.3499 & 0.1429 \\
-0.6547 & 0.3780 & 0.9258 \\
0.2857 & 0.9897 & -0.4041
\end{array}\right]
$$

which yields to the same solution as above. This method to get a basis for the tangent space is faster than the previous one, but we require a valid trivial parameterization.

Finally, we can also get the translation equations directly from the rotations as described in Section 4.3, without having to calculate the directions of the bars. The parametric translation equation in Table 3 can also be written as:

$$
\begin{aligned}
& \left(\begin{array}{c}
d_{n-2} \\
d_{n-1} \\
d_{n}
\end{array}\right)=\mathbf{L}\left(\phi_{n-1}\right)\left(\mathbf{A}_{n-3}^{n-2}(\boldsymbol{\phi})\right)^{t}\left[\left(\begin{array}{c}
d_{n-3} \\
0 \\
0
\end{array}\right)+\left(\mathbf{A}_{n-4}^{n-4}(\boldsymbol{\phi})\right)^{t}\left[\left(\begin{array}{c}
d_{n-4} \\
0 \\
0
\end{array}\right)+\cdots+\left(\mathbf{A}_{2}^{2}(\boldsymbol{\phi})\right)^{t}\left(\begin{array}{c}
d_{2} \\
-d_{1} \\
0
\end{array}\right)\right]\right] \\
& \left(\begin{array}{c}
d_{4} \\
d_{5} \\
d_{6}
\end{array}\right)=\mathbf{L}\left(\phi_{5}\right)\left(\mathbf{A}_{3}^{4}(\boldsymbol{\phi})\right)^{t}\left[\left(\begin{array}{c}
d_{3} \\
0 \\
0
\end{array}\right)+\left(\mathbf{A}_{2}^{2}(\boldsymbol{\phi})\right)^{t}\left(\begin{array}{c}
d_{2} \\
-d_{1} \\
0
\end{array}\right)\right],
\end{aligned}
$$


which leads to

$$
\begin{aligned}
& d_{4}=-0.81 d_{1}-2.16 \\
& d_{5}=-0.65 d_{1}+4.50 \\
& d_{6}=0.29 d_{1}+6.12 .
\end{aligned}
$$

If we set $d_{5}$ to 0 , we obtain the same vector of translations as above. This alternative also requires a trivial parameterization, but not the directions of bars.

\subsubsection{Dealing with a singularity of a trivial parameterization}

Let us take the same trivial parameterization as above, but now we set

$$
\phi_{1}=30^{\circ}, \phi_{2}=0^{\circ}, \phi_{3}=-150^{\circ} \text {. }
$$

Expressing the loop equation as in Eq. (8), we get the following $\mathbf{A}(\boldsymbol{\psi})$ matrix:

$$
\mathbf{A}(\psi)=\left(\begin{array}{ccc}
-1 & 0 & 0 \\
0 & 1 & 0 \\
0 & 0 & -1
\end{array}\right) .
$$

Since $a_{11}=-1$, we are in a singularity of the parameterization; $\left|\begin{array}{lll}\mathbf{n}_{4} & \mathbf{n}_{5} & \mathbf{n}_{6}\end{array}\right|=0$ and $\sin \phi_{5}=0$. In other words, taking the first three rotations, there are infinite solutions for the other three rotations. It can be checked that in this case $\phi_{5}=0$ and $\phi_{6}=\phi_{4}+\pi$.

Now, if we want to calculate a basis for $\mathbf{d}$, we can use the method described in Section 4.1 (Gram-Schmidt) or, alternatively, choose another trivial parameterization (e.g. $\phi_{2}, \phi_{3}$ and $\phi_{4}$ ).

\subsubsection{Dealing with a global singularity}

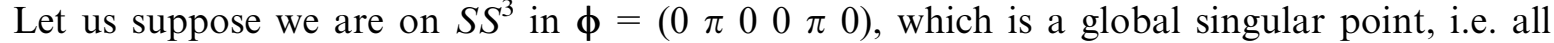
bars are coplanar. This means that we cannot take any parameterization and, therefore, we cannot obtain a basis for $\mathbf{d}$ using the results of Sections 4.2 and 4.3. Instead, we can use the method of Section 4.1 (Gram-Schmidt). The directions of the bars for this point are

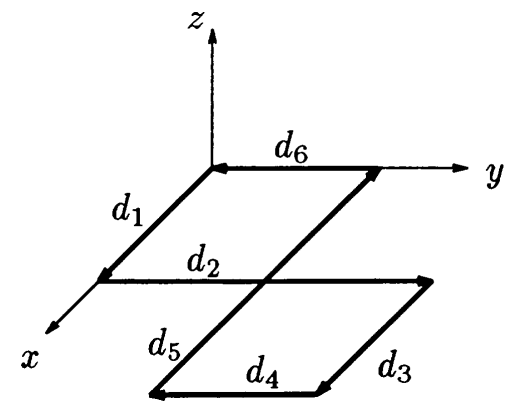

Fig. 8. Singular configuration of the six-bar mechanism. 


$$
\begin{array}{lll}
\mathbf{n}_{1}=\left(\begin{array}{lll}
1 & 0 & 0
\end{array}\right)^{t} & \mathbf{n}_{2}=\left(\begin{array}{lll}
0 & 1 & 0
\end{array}\right)^{t} & \mathbf{n}_{3}=\left(\begin{array}{lll}
1 & 0 & 0
\end{array}\right)^{t} \\
\mathbf{n}_{4}=\left(\begin{array}{llll}
0 & -1 & 1 & 0
\end{array}\right)^{t} & \mathbf{n}_{5}=\left(\begin{array}{lll}
-1 & 0 & 0
\end{array}\right)^{t} & \mathbf{n}_{6}=\left(\begin{array}{lll}
0 & - & 1
\end{array}\right)^{t}
\end{array}
$$

$\mathbf{d}$ has to be orthogonal to $\mathbf{p}_{x}, \mathbf{p}_{y}$ and $\mathbf{p}_{z}$. Now these three vectors are linearly dependent and, therefore, its orthogonal space is of dimension $n-2=4$. Imposing that $\mathbf{d}$ has to be orthogonal to $\mathbf{p}_{x}$ and $\mathbf{p}_{y}$ we obtain

$$
d_{2}=d_{4}+d_{6} \text { and } d_{5}=d_{1}+d_{3},
$$

which is clearly the solution (Fig. 8).

\section{Conclusions}

This paper has expanded on the structure of the self-motion set of the orthogonal spherical mechanism showing how a thorough understanding of it is fundamental in the study of spatial mechanisms. To this end, the following two facts have been exploited: (a) any kinematic loop equation can be modeled as the loop equation derived from the so-called $n$-bar mechanism by taking as many bars as needed and constraining some of the resulting degrees of freedom; and (b) the solution of the translation equation resulting from the factorization of the loop equation of the $n$-bar mechanism is provided by the tangent bundle of the self-motion manifold of its spherical indicatrix. As a consequence, a new unified approach for the analysis of any single kinematic loop containing independent revolute, prismatic and cylindrical pairs has been devised.

\section{References}

[1] Chiang, C. H., Kinematics of Spherical Mechanisms. Cambridge University Press, Cambridge, New York, 1988.

[2] Paul, R. P., Robot Manipulators: Mathematics, Programming and Control. MIT Press, Cambridge, MA, 1985.

[3] Craig, J. J., Introduction to Robotics: Mechanics and Control. Addison-Wesley, Reading, MA, 1989.

[4] Thomas, F., Torras, C., A group-theoretic approach to the computation of symbolic part relations, Journal of Robotics and Automation, 1988, 4(6).

[5] Thomas F. On the $n$-bar mechanism, or how to find global solutions to redundant single loop kinematic chains. IEEE Proceedings of the International Conference for Robotics and Automation 1992;1:403-8.

[6] Hunt, K. H., Kinematic Geometry of Mechanisms. Clarendon Press, Oxford, 1978.

[7] Burdick J W. On the inverse kinematics of redundant manipulators: characterization of the self-motion manifolds. IEEE Proceedings of the International Conference for Robotics and Automation 1989;1:264-70.

[8] Goresky, M., MacPherson, R., Stratified Morse Theory, A Series of Modern Surveys in Mathematics. Springer, Berlin, 1988 .

[9] Warner, F. W., Foundations of differentiable manifolds and Lie groups, in Graduate Texts in Mathematics, 94, ed. I. M. Singer. Massachusetts Institute of Technology, 1971.

[10] Uicker, J. J., Denavit, J., Hartenberg, R. S., An iterative method for the displacement analysis of spatial mechanisms, Journal of Applied Mechanics, Transactions of the ASME, June 1964.

[11] Latombe, J-C., Robot Motion Planning. Kluwer Academic, Dordrecht, 1993.

[12] Thomas, F., Torras, C., Positional inverse kinematic problem in $T^{n} \times \mathbb{R}^{n}$ solved in $T^{2(n+m)}$, in Advances in Robot Kinematics and Computational Geometry, eds. A. J. Lenaričič and B. B. Ravani. Kluwer Academic, Dordrecht, 1994, pp. 291-300. 